\title{
Briófitas do Parque Estadual de Campos do Jordão, Estado de São Paulo, Brasil
}

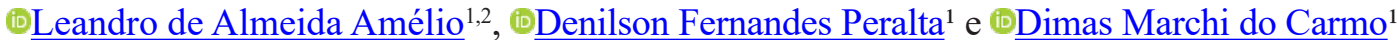

Recebido: 26 outubro 2018; aceito: 14 junho 2019

Como citar: Amélio, L.A., Peralta, D.F. \& Carmo, D.M. 2019. Briófitas do Parque Estadual de Campos do Jordão, Estado de São Paulo, Brasil. Hoehnea 46: e962018. http://dx.doi.org/10.1590/2236-8906-96/2018.

ABSTRACT - (Bryophytes of the Parque Estadual de Campos do Jordão, São Paulo, Brazil). The Parque Estadual de Campos do Jordão (PECJ) is an important remaining area of the Atlantic Forest and does not present bryofloristic surveys. The aim of this study was to survey the bryophyte flora of PECJ and provide data on the diversity of this group of plants in the area, highlighting those species that represent new occurrences for the state of São Paulo. The study includes samples collected from the late 1960s to the end of 2017. As a result, 490 species were recorded, of which 63 are endemic to Brazil and 26 are new records for the state of São Paulo. The most diverse moss families are Orthotrichaceae (22 spp.), Bryaceae (21 spp.), Pottiaceae (21 spp.), and Fissidentaceae (20 spp.). Regarding the liverworts, Lejeuneaceae (80 spp.) is the largest family found in the PECJ.

Keywords: Atlantic forest, conservation unit, floristic survey, new occurrences.

RESUMO - (Briófitas do Parque Estadual de Campos do Jordão, Estado de São Paulo, Brasil). Parque Estadual de Campos do Jordão é uma importante área remanescente da Mata Atlântica e não apresenta levantamentos brioflorísticos. O objetivo deste estudo foi realizar o levantamento da flora de briófitas do PECJParque e, desta forma, fornecer dados sobre a diversidade desse grupo vegetalina área, destacando aquelas espécies que representam novas ocorrências para o Estado de São Paulo. O estudo emgloba amostras coletadas desde o final da década de 1960 até o final de 2017. Como resultado, este estudo apresenta 490 espécies, das quais dessas 63 são endêmicas do Brasil e 26 são novas ocorrências para o Estado de São Paulo. As famílias de musgos mais diversas na área são Orthotrichaceae (22 spp.), Bryaceae (21 spp.), Pottiaceae (21 spp.) e Fissidentaceae (20 spp.). Com relação às hepáticas, Lejeuneaceae (80 spp.) é a maior família encontrada no PECJ. Palavras chaves: levantamento florístico, Mata Atlântica, novas ocorrências, unidade de conservação

\section{Introdução}

Em 26 de junho de 1984, através da Lei Estadual No 4.105 o Parque Estadual de Campos do Jordão (PECJ) foi declarado como a primeira Unidade de Conservação (UC) criada no Estado de São Paulo (1941), uma Área de Proteção Ambiental (APA) e a primeira do país a possuir um Plano de Manejo (1975), um marco histórico no planejamento de áreas protegidas e conservação da biodiversidade. O PECJ apresenta uma cobertura vegetal de transição entre a Mata Atlântica e a Mata de Araucárias. Seu relevo, ligado à altitude, à vegetação e especialmente ao clima local, constitui um grupo de grande valor cênico e biológico. Por causa de sua posição geográfica, situada entre os dois maiores centros urbanos do Brasil, São
Paulo e Rio de Janeiro, a APA tem um considerável potencial turístico e ecológico (SOS Mata Atlântica 2007), que são as atividades econômicas da região.

O Parque Estadual de Campos do Jordão (PECJ) está situado no domínio fitogeográfico da Mata Atlântica, o qual apresenta as maiores taxas de endemismo e de riqueza de briófitas para o país (Costa et al. 2011). Por conta disso, ela é considerada um hotspot mundial que abriga hoje cerca de $12,5 \%$ dos remanescentes florestais e fragmentos de floresta nativa (Fundação SOS Mata Atlântica 2017). A região do PECJ é formada basicamente por um mosaico com três fisionomias básicas: a mata de Araucária e Podocarpus, os Campos de Altitude e a Mata Nebular (Jarenkow \& Batista 1987).

O Estado de São Paulo dispõe de uma série de trabalhos com briófitas ocorrentes em regiões

1. Instituto de Botânica, Núcleo de Pesquisa em Briologia, Avenida Miguel Estéfano, 3687, 04301-902, São Paulo, SP, Brasil

2. Autor para correspodência: ednlora@gmail.com 
da Mata Atlântica, (Visnadi 2005, Visnadi 2006, Yano \& Peralta 2007, Peralta \& Yano 2008, Yano \& Peralta 2008, Visnadi 2009, Peralta \& Yano 2012, Visnadi 2013, Carmo et. al. 2016) que contribuem com o conhecimento da flora regional. Atualmente, 890 espécies ocorrem no domínio da Mata Atlântica refletindo $99 \%$ da brioflora estimada para todo Estado de São Paulo (Costa \& Peralta 2015a).

Até o momento, não há estudos de briófitas realizados para o PECJ, o que se conhece da brioflora dessa região é proveniente de citações para o Estado de São Paulo em bibliografias com enfoques florísticos e ecológicos para Mata Atlântica (Costa \& Lima 2005, Santos \& Costa 2008, Costa \& Santos 2009, Santos \& Costa 2010, Costa \& Peralta 2015b, Silva \& Pôrto 2015, Carmo et al. 2016). O objetivo principal deste estudo é apresentar o levantamento de briófitas mostrando a diversidade do grupo na área do Parque Estadual de Campos do Jordão.

\section{Materiais e métodos}

Área de Estudo - O Parque Estadual de Campos do Jordão (PECJ), está localizado ao norte do município de Campos do Jordão, SP, Brasil ( $\left.22^{\circ} 44^{\prime} \mathrm{S} 45^{\circ} 30^{\prime} \mathrm{W}\right)$. A área está inserida no domínio de Floresta Ombrófila Mista, sobre a Serra da Mantiqueira, preservando remanescentes naturais restritos aos fundos de vale, estando os interflúvios predominantemente ocupados por campos naturais (Souza 2008). O PECJ conta com 8341 ha de área, equivalente a um terço do município de Campos do Jordão, o qual faz divisa com o Estado de Minas Gerais. O relevo é fortemente dissecado pela extensa rede de drenagem da Bacia do Alto Sapucaí, com altitudes variando de 1500 a $2000 \mathrm{~m}$ (Almeida 1964). O clima da região é classificado como temperado brando sem estiagem, de acordo com o sistema de classificação de Köppen (Seibert et al. 1975). A precipitação média anual é de 1804 mm, com temperaturas médias variando de $17,5^{\circ} \mathrm{C}$ no mês mais quente (fevereiro) a $11,5^{\circ} \mathrm{C}$ no mês mais frio (junho), não raro com mínimas abaixo de $0{ }^{\circ} \mathrm{C}$ e ocorrência de geadas (Moreira et al. 2006).

Delineamento amostral - $\mathrm{O}$ estudo engloba amostras coletadas em expedições realizadas por diversos coletores de 1964 até 2017. Em 2017 os autores deste estudo realizaram mais duas expedições e as coletas foram feitas em caminhadas livres nas trilhas disponíveis no parque, cobrindo além da área principal, aproximadamente mais dois metros além do extremo das trilhas. As briófitas foram coletadas em todos os tipos de substratos disponíveis e o método utilizado para a coleta, herborização e preservação do material seguiu Gradstein et al. (2001).

As amostras coletadas foram analisadas e incluídas no herbário do Instituto de Botânica "Maria Eneyda Pacheco Kauffman Fidalgo" (SP). Para a identificação das espécies foram usadas as seguintes referências: Frahm (1991), Sharp et al. (1994), Buck (1998), Gradstein et al. (2001), Gradstein \& Costa (2003), Câmara \& Costa (2006), Câmara (2008a, b), Yano \& Peralta (2009), Yano \& Peralta (2011) e Bordin \& Yano (2013). Os sistemas de classificação adotados para as três divisões foram baseados em Kruijer (2002), Crandall-Stotler et al. (2009), Frey \& Stech (2009), Goffinet et al. (2009), Renzaglia et al. (2009), Costa et al. (2011), Söderström et al. (2013a, b), Gradstein (2015), Gradstein \& Ilkiu-Borges (2015), Carvalho-Silva et al. (2017) e Puttick et al. (2018).

A lista das espécies encontradas na área do parque está organizada em uma tabela por ordem alfabética de divisão, família, gênero e espécie (tabela 1). Para a distribuição mundial e dos domínios fitogeográficos brasileiros que as briófitas ocupam, foram adotadas as bibliografias segundo Costa (2010), Costa et al. (2011), Costa \& Peralta (2015), Carmo \& Peralta (2016), Carmo et al. (2016) e a Flora do Brasil (2020, em prep.).

\section{Resultados \& Discussão}

Foram analisadas 3003 exsicatas e identificadas 77 famílias, 189 gêneros e 490 espécies. Destas, três espécies (três famílias) pertencem à divisão Anthocerotophyta, 225 espécies (65 gêneros e 29 famílias) à Marchantiophyta e 262 espécies (124 gêneros e 46 famílias) pertencem à Bryophyta.

As famílias que apresentaram maior diversidade foram Lejeuneaceae (80 spp.), representando a divisão das hepáticas (Marchantiophyta) e na divisão dos musgos (Bryophyta) as famílias que se destacaram pela maior diversidade foram Orthotrichaceae (22 spp.), Bryaceae (21 spp.) e Pottiaceae (21 spp.), seguidas por Fissidentaceae (20 spp.). A diversidade das espécies encontradas para o PECJ representa $54,44 \%$ das briófitas registradas para o Estado de São Paulo, 32,15\% das espécies encontradas para o Brasil e $12,30 \%$ das espécies reconhecidas para a América tropical (Gradstein et al. 2001, Costa \& Peralta 2015a, Flora do Brasil 2020, em prep.) (tabela 2).

A Mata Atlântica pode ser considerada o maior centro de biodiversidade de briófitas no país (Costa \& 
Table 1. List of species occurring in the Parque Estadual de Campos do Jordão (PECJ), Campos do Jordão, São Paulo State, Brazil. Dom. Fito. (Phytogeographic Domain): AM: Amazon; CA: Caatinga; CE: Cerrado; MA: Atlantic Forest; PA: Pampa; PN: Pantanal; Distr. Mundial (Worldwide distribution). *New occurrence for the State of São Paulo. Acronyms of the Brazilian states according to IBGE (2012).

\begin{tabular}{|c|c|c|c|}
\hline Táxons & Distr. Mundial & Dom. Fito. & Voucher \\
\hline \multicolumn{4}{|l|}{ Anthocerotophyta } \\
\hline \multicolumn{4}{|l|}{ Dendrocerotaceae } \\
\hline Nothoceros minarum (Ness) J.C. Villarreal & América tropical & MA & Peralta et al. 9894 \\
\hline \multicolumn{4}{|l|}{ Notothyladaceae } \\
\hline Phaeoceros laevis (L.) Prosk. & Cosmopolita & AM, CE, MA, PA, PN & Vital 1015 \\
\hline \multicolumn{4}{|l|}{ Phymatocerotaceae } \\
\hline $\begin{array}{l}\text { Phymatoceros bulbiculosus (Broth.) } \\
\text { Stotler et al. }\end{array}$ & América tropical & CE, MA, PA & Peralta et al. 22103 \\
\hline \multicolumn{4}{|l|}{ Bryophyta } \\
\hline \multicolumn{4}{|l|}{ Adelotheciaceae } \\
\hline Adelothecium bogotense (Hampe) Mitt. & Neotropical & MA, PA & Peralta et al. 21782 \\
\hline \multicolumn{4}{|l|}{ Amblystegiaceae } \\
\hline Amblystegium varium (Hedw.) Lindb. & Cosmopolita & MA, PA & Peralta 12485 \\
\hline \multicolumn{4}{|l|}{ Archidiaceae } \\
\hline Archidium julicaule Müll. Hal. & Neotropical & $\mathrm{MA}, \mathrm{CA}, \mathrm{PN}$ & Vital 14607 \\
\hline \multicolumn{4}{|l|}{ Bartramiaceae } \\
\hline * Leiomela bartramioides (Hook.) Paris & Pantropical & MA & Peralta et al. 21840 \\
\hline Leiomela piligera (Hampe) Broth. & Neotropical & MA & Peralta et al. 22076 \\
\hline $\begin{array}{l}\text { Philonotis elongata (Dism.) H.A. Crum } \\
\& \text { Steere }\end{array}$ & Neotropical & $\mathrm{AM}, \mathrm{CE}, \mathrm{MA}$ & Yano et al. 15670 \\
\hline Philonotis uncinata (Schwägr.) Brid. & Cosmopolita & $\begin{array}{c}\mathrm{AM}, \mathrm{CA}, \mathrm{CE}, \mathrm{MA} \\
\mathrm{PA}, \mathrm{PN}\end{array}$ & Peralta et al. 21736 \\
\hline \multicolumn{4}{|l|}{ Brachytheciaceae } \\
\hline $\begin{array}{l}\text { Aerolindgia capillacea (Hornsch.) } \\
\text { M. Menzel }\end{array}$ & Pantropical & MA & Peralta \& Soares 12511 \\
\hline Brachythecium poadelphus Müll. Hal. & $\begin{array}{l}\text { Endêmica do Brasil - } \\
\text { SP, RJ, MG }\end{array}$ & MA & Peralta et al. 9596 \\
\hline Brachythecium ruderale (Brid.) W.R. Buck & Pantropical & MA, PN & Peralta et al. 9425 \\
\hline Eurrhynchium clinocarpum (Taylor) Paris & Neotropical & MA, CE & Peralta et al. 12529 \\
\hline Helicodontium capillare (Hedw.) A. Jaeger & Neotropical & $\mathrm{AM}, \mathrm{CE}, \mathrm{MA}, \mathrm{PA}$ & Peralta \& Soares 12631 \\
\hline $\begin{array}{l}\text { Meteoridium remotifolium (Müll. Hal.) } \\
\text { Manuel }\end{array}$ & Neotropical & $\mathrm{AM}, \mathrm{CE}, \mathrm{MA}$ & Peralta et al. 9738 \\
\hline $\begin{array}{l}\text { Palamocladium leskeoides (Hook.) E. } \\
\text { Britton }\end{array}$ & Neotropical & MA & $\begin{array}{l}\text { Schafer-Verwimp \& } \\
\text { Verwimp } 8527\end{array}$ \\
\hline $\begin{array}{l}\text { Platyhypnidium aquaticum (A. Jaeger) } \\
\text { M. Fleisch. }\end{array}$ & Neotropical & AM, MA & Peralta et al. 9734 \\
\hline $\begin{array}{l}\text { *Rhynchostegium scariosum (Taylor) } \\
\text { A. Jaeger }\end{array}$ & Neotropical & CE, MA, PN & Peralta \& Soares 12616 \\
\hline $\begin{array}{l}\text { Rhynchostegium serrulatum (Hedw.) } \\
\text { A. Jaeger }\end{array}$ & Neotropical & MA & Peralta et al. 9729 \\
\hline Squamidium brasiliense Broth. & $\begin{array}{l}\text { América tropical e } \\
\text { África }\end{array}$ & MA & Peralta et al. 9418 \\
\hline
\end{tabular}


Tabela 1 (continuação)

\begin{tabular}{|c|c|c|c|}
\hline Táxons & Distr. Mundial & Dom. Fito. & Voucher \\
\hline Squamidium leucotrichum (Taylor) Broth. & Neotropical & $\mathrm{MA}, \mathrm{AM}, \mathrm{CE}$ & Peralta \& Soares 12572 \\
\hline Squamidium nigricans (Hook.) Broth. & Neotropical & MA & Peralta 81 \\
\hline Zelometeorium patulum (Hedw.) Manuel & Neotropical & AM, CE, MA, PN & Peralta et al. 9781 \\
\hline \multicolumn{4}{|l|}{ Bruchiaceae } \\
\hline Trematodon longicollis Michx. & Neotropical & $\mathrm{AM}, \mathrm{MA}, \mathrm{CE}, \mathrm{PA}$ & Yano et al. 22097 \\
\hline \multicolumn{4}{|l|}{ Bryaceae } \\
\hline Anomobryum conicum (Hornsch.) Broth. & Neotropical & MA & Peralta 12464 p.p. \\
\hline $\begin{array}{l}\text { *Anomobryum julaceum (Schrad. ex } \mathrm{P} . \\
\text { Gaertn. et al.) Schimp. }\end{array}$ & Neotropical & MA & Peralta et al. 21682 \\
\hline Brachymenium acuminatum Harv. & Cosmopolita & MA, CE & Peralta et al. 9504 \\
\hline Brachymenium consimile (Mitt.) A. Jaeger & Neotropical & MA & Peralta et al. 10069 \\
\hline Brachymenium hornschuchianum Mart. & $\begin{array}{c}\text { Endêmica do Brasil - } \\
\text { BA, ES, MG, RJ, SP, } \\
\text { PR, RN, RS }\end{array}$ & MA & Peralta \& Soares 12642 \\
\hline $\begin{array}{l}\text { Brachymenium radiculosum (Schwägr.) } \\
\text { Hampe }\end{array}$ & Neotropical & CE, MA & Vital 853 \\
\hline *Brachymenium regnelii Hampe & $\begin{array}{c}\text { Endêmica do Brasil - } \\
\text { MG, SC, SP }\end{array}$ & MA & $\begin{array}{l}\text { Schafer-Verwimp \& } \\
\text { Verwimp } 11017\end{array}$ \\
\hline Bryum apiculatum Schwägr. & Cosmopolita & $\mathrm{AM}, \mathrm{CA}, \mathrm{CE}, \mathrm{MA}$ & Peralta et al. 9500 \\
\hline Bryum argenteum Broth. & Cosmopolita & AM, CA, CE, MA, PA & Yano \& Marcelli 19526 \\
\hline Bryum caespiticium Hedw. & Cosmopolita & MA & Peralta 12493 \\
\hline Bryum coronatum Hedw. & Cosmopolita & $\mathrm{AM}, \mathrm{CE}, \mathrm{MA}$ & Peralta et al. 9532 \\
\hline Bryum dichotomum Hedw. & Cosmopolita & MA, CE & Peralta 12498 \\
\hline Bryum huillense Welw. \& Duby & Cosmopolita & MA & Peralta et al. 9862 \\
\hline Bryum limbatum Müll. Hal. & Neotropical & $\mathrm{MA}, \mathrm{CE}$ & Yano \& Marcelli 19392 \\
\hline Rhodobryum beyrichianum (Hornsch.) Paris & Cosmopolita & $\mathrm{AM}, \mathrm{MA}, \mathrm{CE}$ & Peralta 199 \\
\hline $\begin{array}{l}\text { Rhodobryum grandifolium (J. Taylor) } \\
\text { Schimp. }\end{array}$ & Pantropical & MA & Peralta et al. 21766 \\
\hline Rhodobryum roseum (Hedw.) Limpr. & Neotropical & MA & Peralta et al. 21687 \\
\hline Rhodobryum subverticillatum Broth. & Neotropical & MA, AM & Peralta et al. 9689 \\
\hline Rosulabryum billarderi (Schwägr.) Spence & Pantropical & MA, AM, PN, PA, CE & \\
\hline Rosulabryum densifolium (Brid.) Ochyra & Pantropical & MA, CE & Peralta 12514 \\
\hline $\begin{array}{l}\text { Rosulabryum pseudocapillare (Besch.) } \\
\text { Ochyra }\end{array}$ & Pantropical & MA, CE & Peralta et al. 9681 \\
\hline \multicolumn{4}{|l|}{ Calymperaceae } \\
\hline Syrrhopodon gaudichaudii Mont. & Pantropical & $\mathrm{AM}, \mathrm{CA}, \mathrm{CE}, \mathrm{MA}, \mathrm{PN}$ & Peralta et al. 21830 \\
\hline $\begin{array}{l}\text { Syrrhopodon lycopodioides (Brid.) Müll. } \\
\text { Hal. }\end{array}$ & Neotropical & MA & Yano \& Marcelli 19641 \\
\hline Syrrhopodon parasiticus (Brid.) Besch. & Pantropical & AM, CE, MA, PN & Peraltaa et al. 10038 \\
\hline Syrrhopodon prolifer Schwägr. & Pantropical & $\mathrm{AM}, \mathrm{CA}, \mathrm{CE}, \mathrm{MA}$ & Peralta 9941 \\
\hline \multicolumn{4}{|l|}{ Catagoniaceae } \\
\hline $\begin{array}{l}\text { *Catagonium nitidum (Hook. f. \& Wilson) } \\
\text { Broth. }\end{array}$ & Neotropical & $\mathrm{AM}$ & Peralta et al. 9924 \\
\hline
\end{tabular}


Tabela 1 (continuação)

\begin{tabular}{|c|c|c|c|}
\hline Táxons & Distr. Mundial & Dom. Fito. & Voucher \\
\hline \multicolumn{4}{|l|}{ Cryphaeaceae } \\
\hline Cryphaea filiformis (Hedw.) Brid. & Neotropical & MA & Bookerman s.n. (SP135941) \\
\hline Cryphaea rhacomitrioides Müll. Hal. & Neotropical & MA & Peralta et al. 21666 \\
\hline $\begin{array}{l}\text { Schoenobryum concavifolium (Griff.) } \\
\text { Gangulee }\end{array}$ & Cosmopolita & $\mathrm{AM}, \mathrm{CE}, \mathrm{MA}, \mathrm{PA}, \mathrm{PN}$ & Peralta et al. 9577 \\
\hline \multicolumn{4}{|l|}{ Daltoniaceae } \\
\hline Calyptrochaeta setigera (Mitt.) W. R. Buck & Neotropical & MA, AM & Peralta et al. 9886 \\
\hline Daltonia gracilis Mitt. & Neotropical & MA & Peralta et al. 10063 \\
\hline Daltonia marginata Griff. & $\begin{array}{l}\text { Endêmica do Brasil - } \\
\text { SP, RJ }\end{array}$ & MA & Perlta et al. 12553 p.p. \\
\hline Daltonia splachnoides (Sm.) Hook. \& Taylor & Neotropical & MA & Peralta et al. 9581 p.p. \\
\hline \multicolumn{4}{|l|}{ Dicranaceae } \\
\hline Dicranella guilleminiana (Mont.) Mitt. & Neotropical & MA & Peralta et al. 21669 \\
\hline Dicranella fusca Brot. & Neotropical & MA & Peralta et al. 21865 \\
\hline Holomitrium arboreum Mitt. & Neotropical & $\mathrm{MA}, \mathrm{AM}, \mathrm{CE}$ & Buck 26303 \\
\hline Holomitrium crispulum Mart. & Neotropical & $\mathrm{AM}, \mathrm{CE}, \mathrm{MA}, \mathrm{PN}$ & Hoehne 663 \\
\hline Holomitrium nitidum Herzog & $\begin{array}{l}\text { Endêmica do Brasil - } \\
\text { ES, MG, PR, RJ, SC, } \\
\text { SP }\end{array}$ & MA & Yano \& Marcelli 19679 \\
\hline Holomitrium olfersianum Hornsch. & Endêmica do Brasil & MA & Buck 26277 \\
\hline Leucoloma triforme (Mitt.) A. Jaeger & $\begin{array}{l}\text { Endêmica do Brasil - } \\
\text { ES, PR, RJ, SP }\end{array}$ & MA & Peralta et al. 9926 \\
\hline $\begin{array}{l}\text { Microcampylopus curvisetus (Hampe) Giese } \\
\text { \& J.-P. Frahm }\end{array}$ & Neotrópico & $\mathrm{MA}, \mathrm{AM}$ & Peralta \& Soares 12506 \\
\hline \multicolumn{4}{|l|}{ Ditrichaceae } \\
\hline Ceratodon purpureus (Hedw.) Brid. & Neotropical & MA & Peralta et al. 10060 \\
\hline Cladastomum ulei Müll. Hal. & Neotropical & MA & Peralta et al. 10029 \\
\hline *Ditrichum crinale (Taylor) Kuntze & Neotropical & MA & Peralta et al. 22114 \\
\hline Ditrichum paulense Geh. ex Hampe & $\begin{array}{l}\text { Endêmica do Brasil - } \\
\text { PR, RS, SC, SP }\end{array}$ & MA & Peralta et al. 22102 \\
\hline Rhamphidium dicranoides (Müll. Hal.) Paris & Neotropical & MA & Peralta \& Soares 12507 \\
\hline \multicolumn{4}{|l|}{ Entodontaceae } \\
\hline Entodon jamesonii (Taylor) Mitt. & Neotropical & $\mathrm{MA}, \mathrm{CE}$ & Peralta 85 \\
\hline *Entodon virens (Hook. f. \& Wilson) Mitt. & $\begin{array}{l}\text { Endêmica do Brasil - } \\
\text { RJ, SP }\end{array}$ & MA & $\begin{array}{l}\text { Schafer-Verwimp \& } \\
\text { Verwimp } 11043\end{array}$ \\
\hline Erythrodontium longisetum (Hook.) Paris & Neotropical & MA, CE, PN & Yano \& Marcelli 19433 \\
\hline Erythrodontium squarrosum (Hampe) Paris & Neotropical & AM, MA, CE, PN & Bockerman s.n. (SP135958) \\
\hline \multicolumn{4}{|l|}{ Fabroniaceae } \\
\hline Dimerodontium balansae Müll. Hal. & Neotropical & MA & Peralta 12484 p.p. \\
\hline Dimerodontium mendozense Mitt. & Neotropical & MA & Peralta 12489 p.p. \\
\hline Fabronia ciliaris (Brid.) Brid. & $\begin{array}{l}\text { Américas, Havaí e } \\
\text { Japão }\end{array}$ & AM, MA, CA, CE, PN & Peralta 12489 p.p. \\
\hline \multicolumn{4}{|l|}{ Fissidentaceae } \\
\hline Fissidens anguste-limbatus Mitt. & Neotropical & MA, CE, AM & Peralta et al. 12584 \\
\hline Fissidens asplenioides Hedw. & Pantropical & $\mathrm{MA}, \mathrm{CE}$ & Peralta et al. 21751 \\
\hline
\end{tabular}


Tabela 1 (continuação)

\begin{tabular}{|c|c|c|c|}
\hline Táxons & Distr. Mundial & Dom. Fito. & Voucher \\
\hline Fissidens bryoides Hedw. & Neotropical & MA, AM & Peralta et al. 9538 \\
\hline Fissidens crispus Mont. & Neotropical & MA, PN, CE, AM, CA & Peralta et al. 9543 \\
\hline Fissidens curvatus Hornsch. & Neotropical & $\mathrm{MA}, \mathrm{CE}$ & Peralta et al. 9928 \\
\hline Fissidens elegans Brid. & Neotropical & $\begin{array}{l}\text { AM, CA, CE, MA, } \\
\text { PA, PN }\end{array}$ & Peralta et al. 9913 \\
\hline Fissidens hornschuchii Mont. & Neotropical & $\begin{array}{l}\text { AM, CA, CE, MA, } \\
\text { PA, PN }\end{array}$ & Peralta et al. 10031 \\
\hline $\begin{array}{l}\text { Fissidens intromarginatus (Hampe) } \\
\text { A. Jaeger }\end{array}$ & Neotropical & $\mathrm{AM}, \mathrm{MA}, \mathrm{CA}, \mathrm{CE}, \mathrm{PN}$ & Peralta et al. 10051 \\
\hline Fissidens lagenarius Mitt. & Neotropical & $\mathrm{AM}, \mathrm{CA}, \mathrm{CE}, \mathrm{MA}, \mathrm{PN}$ & Vital 9635 \\
\hline Fissidens oediloma Müll. Hal. ex Broth. & Neotropical & MA & Peralta et al. 22071 \\
\hline Fissidens pellucidus Hornsch. & Neotropical & $\begin{array}{c}\text { AM, CA, CE, MA, } \\
\text { PA, PN }\end{array}$ & Peralta 9802 \\
\hline Fissidens rigidulus Hook. f. \& Wilson & Neotropical & MA, CE, PN & Yano 1165 \\
\hline Fissidens saprophilus Broth. & Neotropical & $\mathrm{MA}, \mathrm{CE}$ & Yano \& Prado 26033 \\
\hline Fissidens scariosus Mitt. & Neotropical & $\mathrm{AM}, \mathrm{CE}, \mathrm{MA}, \mathrm{CA}$ & Buck 26264 \\
\hline Fissidens spuriolimbatus Broth. & Neotropical & $\mathrm{MA}, \mathrm{CE}$ & Peralta et al. 9505 \\
\hline Fissidens submarginatus Bruck & Pantropical & $\mathrm{AM}, \mathrm{MA}, \mathrm{CE}, \mathrm{CA}$ & Peralta et al. 9789 \\
\hline Fissidens termitarum (Herzog) Pursell & Neotropical & $\mathrm{MA}, \mathrm{CA}, \mathrm{CE}$ & Peralta et al. 9975 \\
\hline Fissidens yanoae Pursell & $\begin{array}{l}\text { Endêmica do Brasil - } \\
\text { RS, SP }\end{array}$ & MA & Peralta et al. 10015 \\
\hline Fissidens weirii Mitt. & Neotropical & CE, MA & Peralta et al. 9790 \\
\hline Fissidens zollingeri Mont. & Pantropical & $\mathrm{AM}, \mathrm{MA}, \mathrm{CA}, \mathrm{CE}, \mathrm{PN}$ & Peralta et al. 9465 \\
\hline \multicolumn{4}{|l|}{ Funariaceae } \\
\hline Enthostodon bonplandii (Hook.) Mitt. & Neotropical & MA, CE & Peralta et al. 12618 \\
\hline Enthostodon puiggari Gehb. \& Hampe & $\begin{array}{l}\text { Endêmica do Brasil: } \\
\text { RS, SP }\end{array}$ & MA & Yano \& Marcelli 22094 \\
\hline $\begin{array}{l}\text { Enthostodon ramulosus (Hampe) M.S. Dias } \\
\text { \& D.F. Peralta }\end{array}$ & $\begin{array}{l}\text { Endêmica do Brasil: } \\
\text { PR, RS, RJ, SC }\end{array}$ & MA & Peralta et al. 21839 \\
\hline Funaria calvescens Schwagr. & Pantropical & $\mathrm{AM}, \mathrm{MA}, \mathrm{CE}, \mathrm{PN}$ & Peralta 12480 \\
\hline \multicolumn{4}{|l|}{ Hedwigiaceae } \\
\hline $\begin{array}{l}\text { Hedwigidium integrifolium (P. Beauv.) } \\
\text { Dixon }\end{array}$ & Neotropical & MA & Yano \& Prado 26145 \\
\hline \multicolumn{4}{|l|}{ Hookeriaceae } \\
\hline $\begin{array}{l}\text { Hookeria acutifolia Hook. \& Grev. } \\
\text { Hypnaceae }\end{array}$ & Neotropical & MA & Peralta et al. 9994 \\
\hline Calliergonella cuspidata (Hedw.) Loeske & Pantropical & MA & Peralta et al. 9521 \\
\hline Chrysohypnum diminutivum (Hampe) Buck & Cosmopolita & $\mathrm{AM}, \mathrm{CE}, \mathrm{MA}, \mathrm{PA}, \mathrm{PN}$ & Vital 9711 \\
\hline Chrysohypnum elegantulum (Hook.) Hampe & Neotropical & $\mathrm{AM}, \mathrm{CE}, \mathrm{MA}, \mathrm{PN}$ & Peralta 190 \\
\hline Mittenothamnium reduncum (Mitt.) Ochyra & Pantropical & MA & Peralta et al. 21981 \\
\hline $\begin{array}{l}\text { Mittenothamnium reptans (Hampe) Cardot } \\
\text { Hypopterygiaceae }\end{array}$ & Neotropical & CE, MA, PA & Peralta et al. 21889 \\
\hline $\begin{array}{l}\text { Hypopterygium tamariscina (Hedw.) Brid. } \\
\text { ex Müll. Hal. }\end{array}$ & Cosmopolita & MA & Peralta et al. 9904 \\
\hline
\end{tabular}


Tabela 1 (continuação)

\begin{tabular}{|c|c|c|c|}
\hline Táxons & Distr. Mundial & Dom. Fito. & Voucher \\
\hline Lopidium concinnum (Hook.) Wilson & Neotropical & AM, MA & Peralta 9911 \\
\hline \multicolumn{4}{|l|}{ Lembophyllaceae } \\
\hline $\begin{array}{l}\text { Orthostichella pachygastrella (Müll. Hal.) } \\
\text { B.H. Allen \& Magill }\end{array}$ & Neotropical & MA & Peralta et al. 9620 \\
\hline $\begin{array}{l}\text { Orthostichella versicolor (Müll.Hal.) B.H. } \\
\text { Allen \& W. R. Buck }\end{array}$ & Neotropical & AM, MA & Vital 9706 \\
\hline Pilotrichella flexilis (Hedw.) Ångström & Cosmopolita & $\mathrm{AM}, \mathrm{MA}, \mathrm{CE}, \mathrm{PN}$ & Vital 9627 \\
\hline \multicolumn{4}{|l|}{ Lepyrodontaceae } \\
\hline Lepyrodon tomentosus (Hook.) Mitt. & Neotropical & MA & Peralta et al. 21841 \\
\hline \multicolumn{4}{|l|}{ Leskeaceae } \\
\hline Rauiella lagoensis (Hampe) W.R. Buck & Neotropical & CE, MA & Vital 1312 \\
\hline \multicolumn{4}{|l|}{ Leucobryaceae } \\
\hline Campylopus aemulans (Hampe) A. Jaeger & Neotropical & $\mathrm{MA}, \mathrm{CE}$ & Yano et al. 22078 \\
\hline Campylopus arctocarpus (Hornsch.) Mitt. & Pantropical & CA, CE, MA, PA & Peralta 88 \\
\hline Campylopus carolinae Grout & Américas & $\mathrm{AM}, \mathrm{MA}, \mathrm{CE}, \mathrm{PN}$ & Peralta et al. 9990 \\
\hline Campylopus cryptopodioides Broth. & Neotropical & $\mathrm{AM}, \mathrm{MA}, \mathrm{CE}, \mathrm{PN}$ & Yano \& Prado 26096 \\
\hline Campylopus dichrostis (Müll. Hal.) Paris & $\begin{array}{c}\text { Endêmica do Brasil - } \\
\text { BA, GO, MG, RJ, RS, } \\
\text { SC, SP }\end{array}$ & MA, CE & Gibertoni 62 \\
\hline Campylopus filifolius (Hornsch.) Mitt. & Neotropical & $\mathrm{AM}, \mathrm{CA}, \mathrm{MA}$ & Yano \& Marcelli 19689 \\
\hline Campylopus gemmatus (Müll. Hal.) Paris & $\begin{array}{l}\text { Endêmica do Brasil - } \\
\text { DF, MG, PR, RJ, RS }\end{array}$ & MA, CE & Peralta 12488 \\
\hline $\begin{array}{l}\text { Campylopus heterostachys (Hampe) A. } \\
\text { Jaeger }\end{array}$ & Neotropical & $\mathrm{AM}, \mathrm{CA}, \mathrm{CE}, \mathrm{MA}$ & Giancotti 86 \\
\hline Campylopus lamellinervis (Müll. Hal.) Mitt. & Neotropical & CA, MA, PA & Peralta et al. 10058 \\
\hline Campylopus pilifer Brid. & Neotropical & AM, CA, CE, MA, PA & Peralta 182 \\
\hline Campylopus savannarum (Müll. Hal.) Mitt. & Pantropical & $\mathrm{AM}, \mathrm{MA}, \mathrm{CA}, \mathrm{CE}, \mathrm{PN}$ & Peralta 71 \\
\hline Campylopus surinamensis Müll. Hal. & Neotropical & $\mathrm{AM}, \mathrm{MA}, \mathrm{CE}, \mathrm{PN}$ & Vital 521 \\
\hline $\begin{array}{l}\text { Leucobryum albidum (Brid. ex P. Beauv.) } \\
\text { Lindb. }\end{array}$ & Cosmopolita & $\mathrm{AM}, \mathrm{MA}, \mathrm{CE}$ & Gibertoni 4 \\
\hline Leucobryum clavatum Hampe & $\begin{array}{l}\text { Endêmica do Brasil } \\
\text { - BA, DF, GO, MG, } \\
\text { MT, PR, RJ, RS, SC, } \\
\text { SP }\end{array}$ & $\mathrm{MA}, \mathrm{CE}$ & Yano \& Marcelli 19632 \\
\hline Leucobryum crispum Müll. Hal. & Neotropical & $\mathrm{AM}, \mathrm{CE}, \mathrm{MA}$ & Yano et al. 15639 \\
\hline Leucobryum giganteum Müll. Hal. & Neotropical & AM, MA & Peralta et al. 21717 \\
\hline Pilopogon laevis (Taylor) Thér. & Neotropical & MA & Peralta et al. 22083 \\
\hline Leucomiaceae & & & \\
\hline $\begin{array}{l}\text { Philophyllum tenuifolium (Mitt.) Broth. } \\
\text { Meteoriaceae }\end{array}$ & Neotropical & MA & Yano et al. 22080 \\
\hline Floribundaria flaccida (Mitt.) Broth. & América do Sul & $\mathrm{AM}, \mathrm{CE}, \mathrm{MA}, \mathrm{PN}$ & Vital 9700 \\
\hline Meteorium deppei (Hornsch.) Mitt. & Neotropico & MA, CE, PN & $\begin{array}{l}\text { Schafer-Verwimp \& } \\
\text { Verwimp } 11044\end{array}$ \\
\hline
\end{tabular}


Tabela 1 (continuação)

\begin{tabular}{|c|c|c|c|}
\hline Táxons & Distr. Mundial & Dom. Fito. & Voucher \\
\hline Meteorium latifolium (Lindb.) Broth. & $\begin{array}{l}\text { Endêmica do Brasil - } \\
\text { MG, RJ, SP, PR, RS }\end{array}$ & MA & Peralta et al. 21738 \\
\hline Meteorium pseudoteres W.R. Buck & Neotropical & MA & Peralta et al. 9774 \\
\hline Toloxis imponderosa (Taylor) W.R. Buck & Neotropical & MA & Hoehne 643 \\
\hline \multicolumn{4}{|l|}{ Mniaceae } \\
\hline $\begin{array}{l}\text { Plagiomnium rhynchophorum (Hook.) T.J. } \\
\text { Kop. }\end{array}$ & Cosmopolita & $\mathrm{AM}, \mathrm{CE}, \mathrm{MA}$ & Peralta 9863 \\
\hline Pohlia elongata Hedw. & Neotropical & MA & Peralta 9483 \\
\hline Pohlia humilis (Mont.) Broth. & Neotropical & MA & Peralta et al. 22109 \\
\hline Pohlia tenuifolia (A. Jaeger) Broth. & Neotropical & MA & Peralta et al. 12617 \\
\hline $\begin{array}{l}\text { Schizymenium campylocarpum (Arn. \& } \\
\text { Hook.) Shaw }\end{array}$ & Pantropical & MA & Peralta et al. 9475 \\
\hline \multicolumn{4}{|l|}{ Neckeraceae } \\
\hline Neckera scabridens Müll. Hal. & Neotropical & MA & Peralta et al. 12524 \\
\hline Neckeropsis undulata (Hedw.) Reichardt & Américas & $\mathrm{AM}, \mathrm{MA}, \mathrm{CA}, \mathrm{CE}$ & Vital 9699 \\
\hline $\begin{array}{l}\text { Porotrichum korthalsianum (Dozy \& Molk.) } \\
\text { Mitt. }\end{array}$ & Neotropical & MA & Yano \& Marcelli 19696 \\
\hline Porotrichum lancifrons (Hampe) Mitt. & Neotropical & MA & Peralta et al. 21808 \\
\hline Porotrichum longirostre (Hook.) Mitt. & Neotropical & CE, MA & Vital 9660 \\
\hline Porotrichum mutabile Hampe & Pantropical & MA & Hoehne 665 \\
\hline Porotrichum thieleanum (Müll.Hal.) Mitt. & $\begin{array}{l}\text { Endêmica do Brasil - } \\
\text { MG, PR, RJ, RS, SP }\end{array}$ & MA & Peralta et al. 9878 \\
\hline \multicolumn{4}{|l|}{ Orthodontaceae } \\
\hline $\begin{array}{l}\text { Orthodontium pellucens (Hook.) Bruch \& } \\
\text { Schimp. }\end{array}$ & Neotropical & MA & Peralta et al. 21781 \\
\hline \multicolumn{4}{|l|}{ Orthotrichaceae } \\
\hline $\begin{array}{l}\text { Groutiella tomentosa (Hornsch.) Wijk \& } \\
\text { Margad. }\end{array}$ & Cosmopolita & $\mathrm{AM}, \mathrm{MA}, \mathrm{CE}$ & Buck 26278 p.p. \\
\hline Macrocoma tenuis (Hook. \& Grev.) Vitt & $\begin{array}{l}\text { Américas, Havaí e } \\
\text { Japão }\end{array}$ & CE, MA & Peralta et al. 22098 \\
\hline Macromitrium catharinense Paris & Neotropical & MA & Peralta et al. 22086 \\
\hline Macromitrium cirrosum (Hedw.) Brid. & Neotropical & AM, MA & Peralta et al. 21785 \\
\hline Macromitrium contextum Hampe & Neotropical & $\mathrm{AM}, \mathrm{MA}, \mathrm{CE}$ & Buck 26266 \\
\hline Macromitrium guatemalense Müll. Hal. & Neotropical & $\mathrm{AM}, \mathrm{MA}, \mathrm{CE}$ & Peralta et al. 21853 \\
\hline $\begin{array}{l}\text { Macromitrium microstomum (Hook. } \\
\text { \& Grev.) Schwägr. }\end{array}$ & Cosmopolita & MA & Peralta et al. 21856 \\
\hline Macromitrium podocarpi Müll. Hal. & Neotropical & $\mathrm{AM}, \mathrm{MA}, \mathrm{CE}$ & Peralta et al. 21838 \\
\hline $\begin{array}{l}\text { Macromitrium punctatum (Hook. \& Grev.) } \\
\text { Brid. }\end{array}$ & Neotropical & $\mathrm{AM}, \mathrm{CA}, \mathrm{CE}, \mathrm{MA}$ & Peralta et al. 21790 \\
\hline Macromitrium richardii Schwägr. & Neotropical e África & AM, MA & Buck 26312 \\
\hline Schlotheimia apressifolia Mitt. & Neotropical & MA & Peralta et al. 21778 \\
\hline Schlotheimia capillaris Hampe & $\begin{array}{c}\text { Endêmica do Brasil - } \\
\text { RJ, RS }\end{array}$ & MA & Peralta et al. 9495 \\
\hline Schlotheimia elata Mitt. & $\begin{array}{c}\text { Endêmica do Brasil - } \\
\text { RJ, SP }\end{array}$ & MA & Peralta et al. 9510 \\
\hline
\end{tabular}


Tabela 1 (continuação)

\begin{tabular}{|c|c|c|c|}
\hline Táxons & Distr. Mundial & Dom. Fito. & Voucher \\
\hline Schlotheimia jamesonii (Arn.) Brid. & Neotropical & $\mathrm{AM}, \mathrm{MA}, \mathrm{CE}, \mathrm{PA}$ & Vital 9581 \\
\hline Schlotheimia merkelii Hornsch. & $\begin{array}{l}\text { Endêmica do Brasil } \\
\text { - MG }\end{array}$ & MA & Buck 26313 \\
\hline Schlotheimia rugifolia (Hook.) Schwägr. & Neotropical e Índia & $\mathrm{AM}, \mathrm{CE}, \mathrm{MA}$ & Peralta et al. 10019 \\
\hline Schlotheimia tecta Hook. \& Wilson & Neotropical e México & MA & Giancotti 83 \\
\hline Schlotheimia torquata (Hedw.) Brid. & Neotropical & AM, MA & Peralta et al. 22035 \\
\hline Schlotheimia trichomitria Schwägr. & Neotropical & $\mathrm{MA}, \mathrm{CE}$ & Peralta et al. 21709 \\
\hline Zygodon obtusifolius Hook. & Neotropical & MA & Peralta et al. 9506 \\
\hline Zygodon viridissimus (Dicks.) Brid. & Neotropical & MA & Peralta et al. 9563 \\
\hline Zygodon reiwardt (Hornsch.) Braun & Neotropical & MA & Peralta et al. 9755 \\
\hline \multicolumn{4}{|l|}{ Phyllogoniaceae } \\
\hline Phyllogonium viride Brid. & Neotropical e África & MA & Peralta 76 \\
\hline \multicolumn{4}{|l|}{ Pilotrichaceae } \\
\hline Cyclodictyon albicans (Hedw.) Kuntze & Neotropical & MA, CE & Peralta et al. 9883 \\
\hline Cyclodictyon olfersianum (Hornsch.) Kuntze & Neotropical & MA & Yano 1161 \\
\hline Cyclodictyon varians (Sull.) Kuntze & Américas & $\mathrm{AM}, \mathrm{MA}, \mathrm{CE}$ & Peralta et al. 9895 p.p. \\
\hline $\begin{array}{l}\text { Hypnella pilifera (Hook. \& Wilson) } \\
\text { A. Jaeger }\end{array}$ & Neotropical & MA & Peralta 207 \\
\hline Lepidopilidium brevisetum (Hampe) Broth. & Neotropical & MA & Yano \& Prado 26022 \\
\hline Lepidopilum brevipes Mitt. & Neotropical & $\mathrm{AM}, \mathrm{MA}$ & Peralta et al. 21774 \\
\hline Lepidopilum caudicaule (Müll. Hal.) Broth. & América tropical & MA & Peralta \& Soares 12543 p.p. \\
\hline Lepidopilum longifolium Hampe & Neotropical & MA & Yano et al. 22115 \\
\hline Lepidopilum muelleri (Hampe) Mitt. & Neotropical & MA & Peralta et al. 9460 \\
\hline Lepidopilum subsubulatum Geh. \& Hampe & Pantropical & MA & Peralta \& Soares 14217 \\
\hline Thamniopsis incurva (Hornsch.) W.R. Buck & Neotropical & AM, MA & Yano 1160 p.p. \\
\hline $\begin{array}{l}\text { Trachyxiphium guadeloupense (Brid.) W. } \\
\text { R. Buck }\end{array}$ & Neotropical & MA & Peralta et al. 9909 \\
\hline \multicolumn{4}{|l|}{ Plagiotheciaceae } \\
\hline $\begin{array}{l}\text { Plagiothecium novogranatense (Hampe) } \\
\text { Mitt. }\end{array}$ & Neotropical & MA & Peralta et al. 9948 \\
\hline \multicolumn{4}{|l|}{ Polytrichaceae } \\
\hline Atrichum androgynum (Müll. Hal.) A. Jaeger & Neotropical & MA & Peralta et al. 9560 \\
\hline Itatiella ulei (Broth. ex Müll. Hal.) G.L. Sm. & $\begin{array}{l}\text { Endêmica do Brasil - } \\
\text { ES, MG, PR, RJ, SP }\end{array}$ & MA & Peralta et al. 22106 \\
\hline $\begin{array}{l}\text { Itatiella riedeliana (Mont.) N.E. Bell } \\
\text { \& Hyvönen }\end{array}$ & $\begin{array}{l}\text { Endêmica do Brasil - } \\
\text { ES, PR, RJ, SP }\end{array}$ & MA & Yano \& Marcelli 15726 \\
\hline $\begin{array}{l}\text { Pogonatum campylocarpum (Müll. Hal.) } \\
\text { Mitt. }\end{array}$ & Neotropical & MA & Peralta 12467 \\
\hline $\begin{array}{l}\text { Pogonatum pensilvanicum (Bartram ex } \\
\text { Hedw.) P. Beauv. }\end{array}$ & Américas & $\mathrm{MA}, \mathrm{CE}$ & Yano \& Prado 26057 \\
\hline $\begin{array}{l}\text { Polytrichadelphus pseudopolytrichum } \\
\text { (Raddi) G.L. Sm. }\end{array}$ & Neotropical & MA & Peralta 69 \\
\hline
\end{tabular}


Tabela 1 (continuação)

\begin{tabular}{|c|c|c|c|}
\hline Táxons & Distr. Mundial & Dom. Fito. & Voucher \\
\hline Polytrichum angustifolium Mitt. & $\begin{array}{l}\text { Endêmica do Brasil - } \\
\text { ES, MG, PR, RS, SC, } \\
\text { SP }\end{array}$ & MA, PA & Yano \& Marcelli 19685 \\
\hline Polytrichum commune L. ex Hedw. & Cosmopolita & $\mathrm{AM}, \mathrm{CE}, \mathrm{MA}$ & Peralta 191 \\
\hline Polytrichum juniperinum Wild. ex Hedw. & Cosmopolita & $\mathrm{AM}, \mathrm{CE}, \mathrm{MA}$ & Hoehne 634 \\
\hline \multicolumn{4}{|l|}{ Pottiaceae } \\
\hline Acaulon uleanum Müll. Hal. & Neotropical & MA, PA & Vital 14606 \\
\hline Barbula indica (Hook.) Spreng. & Pantropical & $\mathrm{AM}, \mathrm{CA}, \mathrm{CE}, \mathrm{MA}, \mathrm{PN}$ & Peralta \& Soares 12555 \\
\hline Chenia leptophylla (Müll. Hal.) R. H. Zander & Neotropical & MA & Peralta 12495 \\
\hline Gymnostomum aeruginosum $\mathrm{Sm}$. & Neotropical & $\mathrm{MA}, \mathrm{CE}$ & Peralta et al. 9476 \\
\hline $\begin{array}{l}\text { Hymenostylium recurvirostrum (Hedw.) } \\
\text { Dixon }\end{array}$ & Neotropical & $\mathrm{MA}, \mathrm{CE}$ & Yano \& Marcelloi 19706 \\
\hline Leptodontium araucarietii (Müll. Hal.) Paris & Neotropical & MA & Yano \& Watanabe 9236 \\
\hline *Leptodontium capituligerum Müll. Hal. & Neotropical & PA & Peralta et al. 9671 \\
\hline Leptodontium pungens (Mitt.) Kindb. & Neotropical & MA, PA & Yano \& Marcelli 19415 \\
\hline $\begin{array}{l}\text { Leptodontium viticulosoides (P. Beauv.) } \\
\text { Wijk \& Margad. }\end{array}$ & Neotropical & $\mathrm{MA}, \mathrm{CE}$ & Peralta et al. 21893 \\
\hline Leptodontium wallisii (Müll.Hal.) Kindb. & Neotropical & MA & Peralta et al. 9450 \\
\hline $\begin{array}{l}\text { Molendoa sendtneriana (Bruch \& Schimp.) } \\
\text { Limpr. }\end{array}$ & Neotropical & $\mathrm{MA}, \mathrm{CE}, \mathrm{PN}$ & Peralta et al. 9684 \\
\hline $\begin{array}{l}\text { Oxystegus tenuirostris (Hook. \& Taylor) } \\
\text { A.J.E. Sm. }\end{array}$ & Neotropical & MA, CE & Peralta et al. 9884 \\
\hline $\begin{array}{l}\text { Pseudosymblepharis schimperiana (Paris) } \\
\text { H. A. Crum }\end{array}$ & Neotropical & MA, CE, PN & Peralta et al. 21877 \\
\hline Streptopogon calymperes Müll. Hal. & Neotropical & MA & Peralta et al. 10030 \\
\hline $\begin{array}{l}\text { Syntrichia amphidiaceae (Müll. Hal.) R. } \\
\text { H. Zander }\end{array}$ & Neotropical & MA, PA & Peralta et al. 12486 \\
\hline Syntrichia laevipila Brid. & Neotropical & MA, PA & Peralta et al. 21711 \\
\hline $\begin{array}{l}\text { Tortella linearis (Sw. ex F. Weber \& D. } \\
\text { Mohr) R. H. Zander }\end{array}$ & Neotropical & MA & Peralta et al. 9451 \\
\hline Tortula muralis Hedw. & Neotropical & MA, CE, PA & Peralta 12482 \\
\hline Trichostomum leptocylindricum Müll. Hal. & Neotropical & $\mathrm{MA}, \mathrm{CE}$ & Peralta et al. 10023 \\
\hline Tuerckheimia guatemalensis Broth. & Neotropical & $\mathrm{MA}, \mathrm{CE}$ & Peralta et al. 10001 \\
\hline Weissia breutelii Müll. Hal. & Neotropical & MA, CE, PA & Vital 680 \\
\hline \multicolumn{4}{|l|}{ Prionodontaceae } \\
\hline \multicolumn{4}{|l|}{ Pterobryaceae } \\
\hline $\begin{array}{l}\text { Calyptothecium duplicatum (Schwägr.) } \\
\text { Broth. }\end{array}$ & Neotropical & MA, CE & Peralta et al. 9936 \\
\hline *Orthostichopsis tijucae (Müll. Hal.) Broth. & $\begin{array}{l}\text { Endêmica do Brasil - } \\
\text { PR, RJ, RS, SC, SP }\end{array}$ & MA & Peralta et al. 9947 \\
\hline Orthostichopsis tortipilis (Müll. Hal.) Broth. & Neotropical & AM, MA & Vital 9720 \\
\hline Pterobryon densum Hornsch. & Neotropical & MA & Peralta et al. 9817 \\
\hline
\end{tabular}


Tabela 1 (continuação)

\begin{tabular}{|c|c|c|c|}
\hline Táxons & Distr. Mundial & Dom. Fito. & Voucher \\
\hline \multicolumn{4}{|l|}{ Ptychomitriaceae } \\
\hline $\begin{array}{l}\text { Ptychomitrium sellowianum (Müll. Hal.) A. } \\
\text { Jaeger }\end{array}$ & Neotropical & MA, PA & Yano \& Marcelli 19531 \\
\hline \multicolumn{4}{|l|}{ Pylaisiadelphaceae } \\
\hline Isopterygium byssobolax (Müll. Hal.) Paris & Pantropical & $\mathrm{AM}, \mathrm{MA}, \mathrm{CE}$ & Peralta et al. 9437 \\
\hline Isopterygium subbrevisetum (Hampe) Broth. & Neotropical & AM, MA & Yano \& Prado 26066 p.p. \\
\hline Isopterygium tenerifolium Mitt. & Neotropical & $\mathrm{AM}, \mathrm{MA}, \mathrm{CE}$ & Peralta et al. 22019 \\
\hline Wijkia flagellifera (Broth.) H.A. Crum & Neotropical & MA & Buck 26280 \\
\hline \multicolumn{4}{|l|}{ Racopilaceae } \\
\hline $\begin{array}{l}\text { Racopilum tomentosum (Hedw.) Brid. } \\
\text { Rhizogoniaceae }\end{array}$ & Cosmopolita & $\mathrm{AM}, \mathrm{CE}, \mathrm{MA}, \mathrm{PA}, \mathrm{PN}$ & Peralta et al. 9935 \\
\hline $\begin{array}{l}\text { Pyrrhobryum spiniforme (Hedw.) Mitt. } \\
\text { Rigodiaceae }\end{array}$ & Cosmopolita & $\mathrm{AM}, \mathrm{CE}, \mathrm{MA}, \mathrm{PA}$ & Peralta et al. 9723 \\
\hline $\begin{array}{l}\text { *Rigodium toxarion (Schwägr.) Schimp. } \\
\text { Sematophyllaceae }\end{array}$ & Neotropical & $\mathrm{AM}, \mathrm{PA}$ & Peralta 100 \\
\hline $\begin{array}{l}\text { Acroporium exiggum (Broth.) W.R. Buck } \\
\text { \& Schäf.-Verw. Buck }\end{array}$ & $\begin{array}{l}\text { Endêmica do Brasil - } \\
\text { MG, RJ, SP }\end{array}$ & MA & Buck 26292 \\
\hline $\begin{array}{l}\text { Aptychopsis estrellae (Müll. Hal.) P.E.A.S. } \\
\text { Câmara et al. }\end{array}$ & Neotropical & $\mathrm{CE}, \mathrm{MA}$ & Peralta et al. 21705 \\
\hline $\begin{array}{l}\text { Aptychopsis pyrrhophylla (Müll.Hal.) Wijk } \\
\text { \& Margad. }\end{array}$ & $\begin{array}{l}\text { Endêmica do Brasil - } \\
\text { AM, BA, MG, RJ ,SP }\end{array}$ & $\mathrm{AM}, \mathrm{MA}$ & Vital 9634 \\
\hline $\begin{array}{l}\text { Aptychopsis tequendamense (Hampe) } \\
\text { P.E.A.S. Câmara et al. }\end{array}$ & Neotropical & MA & Peralt et al. 9446 \\
\hline $\begin{array}{l}\text { Brittonodoxa litophila (Hornsch.) W.R. } \\
\text { Buck et al. }\end{array}$ & Neotropical & $\mathrm{AM}, \mathrm{CE}, \mathrm{MA}$ & Vital 9657 \\
\hline $\begin{array}{l}\text { Brittonodoxa subpinnata (Brid.) W.R. Buck } \\
\text { et al. }\end{array}$ & Pantropical & $\begin{array}{l}\mathrm{AM}, \mathrm{CA}, \mathrm{CE}, \mathrm{MA} \\
\mathrm{PA}, \mathrm{PN}\end{array}$ & Yano \& Marcelli 197713 \\
\hline $\begin{array}{l}\text { Donnellia commutata (Müll. Hal.) W.R. } \\
\text { Buck }\end{array}$ & Neotropical & $\mathrm{AM}, \mathrm{CE}, \mathrm{MA}, \mathrm{PN}$ & Peralta et al. 9478 p.p. \\
\hline Donnellia lagenifera (Mitt.) W. R. Buck & Pantropical & AM, MA & Peralta et al. 9554 \\
\hline $\begin{array}{l}\text { Pterogoniopsis paulista (W.R. Buck \& Vital) } \\
\text { Carv.-Silva et al. }\end{array}$ & $\begin{array}{l}\text { Endêmica do Brasil } \\
\text { - SP }\end{array}$ & MA & Peralta et al. 9487 \\
\hline $\begin{array}{l}\text { Sematophyllum leucostomum (Hampe) W.R. } \\
\text { Buck }\end{array}$ & $\begin{array}{l}\text { Endêmica do Brasil - } \\
\text { PR, RJ, SP }\end{array}$ & MA & Buck 26254 \\
\hline $\begin{array}{l}\text { Sematophyllum subdepresseum (Hampe) } \\
\text { Broth. }\end{array}$ & $\begin{array}{l}\text { Endêmica do Brasil - } \\
\text { GO, MT, PR, RJ, RS, } \\
\text { SC }\end{array}$ & MA & Peralta et al. 21694 \\
\hline $\begin{array}{l}\text { Sematophyllum swartzii (Schwägr.) Welch } \\
\text { \& H.A. Crum }\end{array}$ & Neotropical & MA & Hoehne 644 \\
\hline Trichosteleum glaziovii (Hampe) W.R. Buck & $\begin{array}{l}\text { Endêmica do Brasil - } \\
\text { MG, PE, PR, RJ, RS, } \\
\text { SC, SP }\end{array}$ & MA & Peralta et al. 9615 \\
\hline $\begin{array}{l}\text { Vitalia cuspidiferum (Mitt.) P.E.A.S. Câmara } \\
\text { et al. }\end{array}$ & Neotropical & $\mathrm{CE}, \mathrm{MA}, \mathrm{PN}$ & Peralta et al. 9656 \\
\hline
\end{tabular}


Tabela 1 (continuação)

\begin{tabular}{|c|c|c|c|}
\hline Táxons & Distr. Mundial & Dom. Fito. & Voucher \\
\hline $\begin{array}{l}\text { Vitalia galipensis (Müll. Hal.) P.E.A.S. } \\
\text { Câmara et al. }\end{array}$ & Neotropical & $\mathrm{AM}, \mathrm{CE}, \mathrm{MA}$ & Peralta et al. 9677 \\
\hline \multicolumn{4}{|l|}{ Sphagnaceae } \\
\hline Sphagnum brasiliense Warnst. & $\begin{array}{l}\text { Endêmica do Brasil - } \\
\text { MG, PR, RJ, SP, TO }\end{array}$ & MA & Peralta et al. 9966 \\
\hline *Sphagnum divisum H. A. Crum & $\begin{array}{c}\text { Endêmica do Brasil - } \\
\text { BA, DF, GO, MG, PR, } \\
\text { RJ, SC, SP }\end{array}$ & $\mathrm{MA}, \mathrm{CE}$ & Yano \& Prado 26034 \\
\hline Sphagnum exquisitum H. A. Crum & $\begin{array}{l}\text { Endêmica do Brasil - } \\
\text { ES, MG, PR, RJ, SP }\end{array}$ & MA & Yano et al. 15668 \\
\hline Sphagnum gracilescens Müll. Hal. & $\begin{array}{c}\text { Endêmica do Brasil } \\
\text { - AM, BA, CE, DF, } \\
\text { ES, MG, PR, RJ, RS, } \\
\text { SC, SP }\end{array}$ & $\mathrm{MA}, \mathrm{CE}$ & Peralta et al. 21678 \\
\hline Sphagnum longistolo Müll. Hal. & Pantropical & MA & Yano \& Marcelli 19591 \\
\hline Sphagnum magellanicum Brid. & Cosmopolita & $\mathrm{AM}, \mathrm{CE}, \mathrm{MA}$ & Peralta et al. 9969 \\
\hline Sphagnum pseudoramulinum H. A. Crum & $\begin{array}{l}\text { Endêmica do Brasil - } \\
\text { ES, MG, RJ, RS, SP }\end{array}$ & MA & Yano et al. 15677 \\
\hline Sphagnum ramulinum Warnst. & $\begin{array}{l}\text { Endêmica do Brasil - } \\
\text { BA, DF, GO, MG, RJ }\end{array}$ & $\mathrm{MA}, \mathrm{CE}$ & Peralta et al. 10048 \\
\hline $\begin{array}{l}\text { Sphagnum tenerum Sull. \& Lesq. } \\
\text { Thuidiaceae }\end{array}$ & \multicolumn{2}{|c|}{ Thuidiaceae } & Peralta et al. 9830 \\
\hline Pelekium involvens (Hedw.) Touw & Cosmopolita & AM, CE, MA, PA, PN & Yano \& Prado 26140 \\
\hline Pelekium minutulum (Hedw.) Touw & Neotropical & MA, CE & Peralta et al. 21817 \\
\hline $\begin{array}{l}\text { Thuidiopsis sparsa (Hook. f. \& Wilson) } \\
\text { Broth. }\end{array}$ & Neotropical & MA & Peralta et al. 9466 \\
\hline Thuidium assimile (Mitt.) A. Jaeg. & Neotropical & MA & Bookerman s.n. (SP135956) \\
\hline $\begin{array}{l}\text { Thuidium bifidum A. Soares \& P.E.A.S. } \\
\text { Câmara }\end{array}$ & $\begin{array}{l}\text { Endêmica do Brasil } \\
\text { - GO, MG, RJ, RS, } \\
\text { SC, SP }\end{array}$ & $\mathrm{MA}, \mathrm{CE}$ & Peralta et al. 10041 \\
\hline Thuidium brasilense Mitt. & Neotropical & MA & Buck 26294 \\
\hline Thuidium delicatulum (Hedw.) A. Touw & Cosmopolita & $\mathrm{AM}, \mathrm{CE}, \mathrm{MA}$ & Peralta et al. 9536 \\
\hline $\begin{array}{l}\text { Thuidium pseudoprotensum (Müll. Hal.) } \\
\text { Mitt. }\end{array}$ & Neotropical & MA & Peralta et al. 9916 \\
\hline $\begin{array}{l}\text { Thuidium tamariscinum (Hedw.) Bruch \& } \\
\text { Schimp. }\end{array}$ & Cosmopolita & MA, PA & Buck 26267 \\
\hline Thuidium tomentosum Schimp. & Neotropical & $\mathrm{AM}, \mathrm{CE}, \mathrm{MA}, \mathrm{PN}$ & Vital 9608 \\
\hline \multicolumn{4}{|l|}{ Marchantiophyta } \\
\hline \multicolumn{4}{|l|}{ Acrobolbaceae } \\
\hline $\begin{array}{l}\text { Tylimanthus laxus (Lehm. \& Lindenb.) } \\
\text { Steph. }\end{array}$ & América tropical & CE, MA, PN & Peralta et al. 9805 \\
\hline \multicolumn{4}{|l|}{ Adelanthaceae } \\
\hline *Adelanthus carabayensis (Mont.) Grolle & América tropical & MA & Peralta et al. 9747 \\
\hline
\end{tabular}


Tabela 1 (continuação)

\begin{tabular}{l}
\hline Táxons \\
\hline Aneuraceae \\
Aneura pinguis (L.) Dumort. \\
Riccardia cataractarum (Spruce) Schiffn. \\
Riccardia chamedryfolia (With.) Grolle \\
Riccardia digitiloba (Spruce ex Steph.) \\
Pagán \\
Riccardia fucoides (Sw.) C. Massal. \\
Riccardia glaziovii (Spruce) Meenks \\
Riccardia metzgeriiformis (Steph.) R.M. \\
Schust.
\end{tabular}

Riccardia regnelli (Aongström.) Hell

Riccardia tenuicola (Spruce) Meenks

Balantiopsaceae

Balantiopsis brasiliensis Steph.

Isotachis aubertii (Schwägr.) Mitt.

*Isotachis inflata Steph.

Isotachis multiceps (Lindenb. \& Gottsche) Gottsche

Isotachis serrulata (Sw.) Gottsche

Neesioscyphus argilaceus (Nees) Grolle

Neesioscyphus homophyllus (Nees) Grolle

Calypogeiaceae

Calypogeia grandistipula (Steph.) Steph.

Calypogeia laxa Gottsche \& Lindenb.

Calypogeia peruviana Nees \& Mont.

Mnioloma caespitosa (Spruce) R. M. Schust.

Cephaloziaceae

Odontoschisma denudatum (Nees) Dumort.

Odontoschisma longiflorum (Taylor) Steph.

Odontoschisma variabile (Lindenb. \&

Gottsche) Trevis.

Cephaloziellaceae

Cephaloziella divaricata (Sm.) Schiffn.

*Cephaloziella granatensis (J.B. Jack)

Fulford

Chonecoleaceae

Chonecolea doellingerii (Nees) Grolle
Distr. Mundial

Dom. Fito.

Voucher

Subcosmopolita

América tropical

Pantropicais

AM, MA, PN
MA, CE, PN

MA

Vital 9716

Vital 1762

América tropical

América tropical

Neotropical

Neotropical

AM, CE, MA, PN

MA

AM, MA

AM, MA

Peralta et al. 21743

Peralta et al. 21993

Peralta et al. 21866

Peralta et al. 9977

Peralta et al. 21806

Endêmica do Brasil -

BA, ES, MG, MT, RJ, RS, SC, SP

América do Sul

Endêmica do Brasil ES, MG, RJ, RS, SP

América do Sul e África

Endêmica do Brasil ES, RJ, SC, SP

América tropical

América tropical

Brasil e Peru

Brasil e Argentina

Endêmica do Brasil ES, MG, RJ, RS, SP

América tropical

América tropical

América do Sul

Cosmopolita

América tropical

América tropical

Pantropical

América tropical

América tropical, África, Austrália
MA

Peralta et al. 21886

MA, CE

Peralta et al. 9467

MA, AM

Yano \& Marcelli 19481

MA

MA

MA

Yano \& Prado 26134

AM, MA

MA, CE

MA

MA

AM, MA

AM, CE, MA

AM

Yano \& Marcelli 19588

Vital 9643

Yano \& Prado 26132

Yano \& Marcelli 19444

Peralta et al. 9812

AM, CE, MA

MA, CE

Vital 608

Peralta et al. 9597

AM, CE, MA

Peralta et al. 21802

MA

Peralta et al. 9954

MA

Peralta et al. 10066

MA, CE

Peralta et al. 9808 
Tabela 1 (continuação)

\begin{tabular}{|c|c|c|c|}
\hline Táxons & Distr. Mundial & Dom. Fito. & Voucher \\
\hline \multicolumn{4}{|l|}{ Fossombroniaceae } \\
\hline Fossombronia porphyrorhiza (Nees) Prosk. & Cosmopolita & $\mathrm{MA}, \mathrm{CE}, \mathrm{CA}, \mathrm{PN}$ & Peralta et al. 21972 \\
\hline \multicolumn{4}{|l|}{ Frullaniaceae } \\
\hline Frullania atrata (Sw.) Nees & América tropical & AM, MA & Peralta 91 \\
\hline $\begin{array}{l}\text { Frullania beyrichiana (Lehm. \& Lindenb.) } \\
\text { Lehm. \& Lindenb. }\end{array}$ & América tropical & $\mathrm{AM}, \mathrm{CE}, \mathrm{MA}$ & Peralta et al. 21810 \\
\hline Frullania brasiliensis Raddi & América tropical & MA, CE & Peralta 77 \\
\hline Frullania caulisequa (Nees) Nees & América tropical & $\mathrm{AM}, \mathrm{CA}, \mathrm{CE}, \mathrm{MA}, \mathrm{PA}$ & Peralta et al. 21794 \\
\hline Frullania ecklonii (Spreng.) Gottsche et al. & Pantropical & & Yano et al. 22085 p.p. \\
\hline Frullania ericoides (Nees) Mont. & Pantropical & $\begin{array}{c}\text { AM, CA, CE, MA, } \\
\text { PA, PN }\end{array}$ & Giancotti 49 \\
\hline $\begin{array}{l}\text { Frullania glomerata (Lehm. \& Lindenb.) } \\
\text { Mont. }\end{array}$ & América tropical & $\mathrm{MA}, \mathrm{CA}, \mathrm{CE}, \mathrm{PN}$ & Peralta et al. 9492 \\
\hline Frullania griffithsiana Gottsche & $\begin{array}{l}\text { Endêmica do Brasil - } \\
\text { BA, ES, MG, RS, SP }\end{array}$ & MA & Vital 9617 \\
\hline $\begin{array}{l}\text { Frullania kunzei (Lehm. \& Lindenb.) Lehm. } \\
\text { \& Lindenb. }\end{array}$ & América tropical & $\mathrm{AM}, \mathrm{CE}, \mathrm{MA}, \mathrm{PN}$ & Peralta \& Soares 12519 \\
\hline Frullania obscura (Sw.) Nees ex Mont. & Pantropical & $\mathrm{AM}, \mathrm{CE}, \mathrm{MA}$ & Peralta et al. 9695 \\
\hline Frullania setigera Steph. & América tropical & MA & Giancotti 85 \\
\hline \multicolumn{4}{|l|}{ Geocalycaceae } \\
\hline Saccogynidium caldense (Angstr.) Grolle & $\begin{array}{l}\text { Endêmica do Brasil } \\
\text { - GO, MG, PR, RJ, } \\
\text { SC, SP }\end{array}$ & MA & Peralta et al. 9766 \\
\hline \multicolumn{4}{|l|}{ Arnelliaceae } \\
\hline $\begin{array}{l}\text { Gongylanthus liebmannianus (Lindenb. \& } \\
\text { Gottsche) Steph. }\end{array}$ & América tropical & MA & Peralta et al. 9984 \\
\hline \multicolumn{4}{|l|}{ Herbertaceae } \\
\hline Herbertus bivittatus Spruce & América tropical & AM, MA & Peralta et al. 22028 \\
\hline Herbertus pensilis (Tayl.) Spruce & América tropical & $\mathrm{AM}, \mathrm{MA}$ & Vital 510 \\
\hline Herbertus serratus Spruce & Neotropical & MA & Peralta et al. 9960 \\
\hline \multicolumn{4}{|l|}{ Jamesoniellaceae } \\
\hline $\begin{array}{l}\text { Syzygyella anomala (Lindenb. \& Gottsche) } \\
\text { Steph. }\end{array}$ & Neotropical & MA & Vital 503 \\
\hline Syzygyella concreta (Gottsche) Spruce & América tropical & MA & Peralta et al. 21724 \\
\hline Syzygyella geminifolia (Mitt.) Steph. & América tropical & MA & Peralta et al. 9745 \\
\hline Syzygyella liberata Inoue & América tropical & MA & Vital 499 \\
\hline Syzygyella perfoliata (Sw.) Spruce & América tropical & MA & Peralta et al. 9632 \\
\hline Syzygyella rubricaulis (Nees) Grolle & América tropical & MA & Peralta et al. 21899 \\
\hline Syzygyella sonderi (Gottsche) Feldberg et al. & Neotropical & MA & Vital 1945 \\
\hline *Syzygyella uleana Steph. & Sudeste do Brasil & MA & Peralta et al. 21825 \\
\hline \multicolumn{4}{|l|}{ Jungermanniaceae } \\
\hline Anastrophyllum piligerum (Nees) Steph. & Pantropical & MA & Vital 594 \\
\hline Anastrophyllum tubulosum (Nees) Grolle & América tropical & MA & Peralta et al. 9531 \\
\hline
\end{tabular}


Tabela 1 (continuação)

\begin{tabular}{|c|c|c|c|}
\hline Táxons & Distr. Mundial & Dom. Fito. & Voucher \\
\hline Jungermannia amoena Lindenb. \& Gottsche & América tropical & AM, MA & Vital 500 \\
\hline Jungermannia hyalina Lyell & Cosmopolita & CE, MA, PN & Vital 502 \\
\hline Jungermannia sphaerocarpa Hook. & Cosmopolita & MA & Peralta et al. 9953 \\
\hline \multicolumn{4}{|l|}{ Lejeuneaceae } \\
\hline $\begin{array}{l}\text { Acanthocoleus aberrans (Lindenb. \& } \\
\text { Gottsche) Kruijt }\end{array}$ & América Tropical & CE, MA, PN & Peralta et al. 21780 \\
\hline $\begin{array}{l}\text { Anoplolejeunea conferta (C.F.W. Meissn.) } \\
\text { A. Evans }\end{array}$ & América tropical & AM, MA & Peralta et al. 21842 \\
\hline $\begin{array}{l}\text { *Blepharolejeunea securifolia (Steph.) } \\
\text { R.M.Schust. }\end{array}$ & $\begin{array}{l}\text { América tropical e do } \\
\text { Sul }\end{array}$ & MA & Yano et al. 15751 \\
\hline Brachiolejeunea laxifolia (Taylor) Schiffn. & América tropical & MA & Peralta \& Soares 12523 p.p. \\
\hline $\begin{array}{l}\text { Brachiolejeunea phyllorhiza (Nees) Kruijt } \\
\text { \& Gradst. }\end{array}$ & $\begin{array}{l}\text { América tropical e } \\
\text { África }\end{array}$ & AM, MA & Yano \& Marcelli 19503 p.p. \\
\hline Bryopteris diffusa (Sw.) Nees & América tropical & $\mathrm{AM}, \mathrm{CE}, \mathrm{MA}$ & Bokerman s.n. (SP133218) \\
\hline Bryopteris filicina (Sw.) Nees & Neotropical & AM, CE, MA, PN & Peralta 9932 \\
\hline Ceratolejeunea confusa R.M. Schust. & América tropical & AM, MA & Peralta et al. 22089 \\
\hline Ceratolejeunea cornuta (Lindenb.) Schiffn. & América tropical & AM, MA & Peralta et al. 21820 \\
\hline $\begin{array}{l}\text { Ceratolejeunea fallax (Lehm. \& Lindenb.) } \\
\text { Bonner }\end{array}$ & Neotropical & AM, MA & Peralta et al. 21814 \\
\hline Cheilolejeunea acutangula (Nees) Grolle & Neotropical & $\mathrm{AM}, \mathrm{CE}, \mathrm{MA}$ & Peralta et al. 9544 \\
\hline Cheilolejeunea adnata (Kunze) Grolle & América tropical & $\mathrm{AM}, \mathrm{MA}, \mathrm{PN}$ & Yano \& Marcelli 19567 \\
\hline Cheilolejeunea aneogyna (Spruce) A. Evans & Brasil e Suriname & AM, MA & Peralta et al. 9736 p.p. \\
\hline
\end{tabular}

Cheilolejeunea beyrichii (Lindenb.) Reiner

Cheilolejeunea clausa (Nees \& Mont.) R.M.

Schust.

Cheilolejeunea discoidea (Lehm. \&

Lindenb.) Kachr. \& Schust.

Cheilolejeunea filiformis (Sw.) W. Ye et al.

Cheilolejeunea holostipa (Spruce) Grolle \& R.L. Zhu

Cheilolejeunea insecta Groll

Cheilolejeunea oncophylla (Angstr.) Grolle \& E. Reiner.

Cheilolejeunea unciloba (Lindenb.)

Malombe

Cheilolejeunea xanthocarpa (Lehm. \&

Lindenb.) Malombe

Cololejeunea cardiocarpa (Mont.) A. Evans

Cololejeunea clavatopapilata Steph.

Cololejeunea diaphana A. Evans
Neotropical

América tropical

América tropical

América tropical

América tropical

Endêmica do Brasil MG, RJ, SP

América tropical

América tropical e África

Pantropical

Pantropical

América do Sul,

Flórida e Cuba

Endêmica do Brasil AM, ES, GO, MT, PA, PE, RJ, RS, SC, SP
AM, MA, CA, CE, PN Yano \& Marcelli 19418 p.p.

AM, MA, CE, PN Peralta et al. 9667 p.p.

$\begin{array}{cc}\text { CE, MA, PN } & \text { Peralta et al. } 21807 \\ \text { AM, MA, PN } & \text { Peralta } 79\end{array}$

AM, MA Peralta et al. 10014 p.p.

MA

Schafer-Verwimp 14459 (paratipo)

MA

Yano \& Marcelli 19486

MA, CE

Peralta 86

$\mathrm{MA}, \mathrm{CE}$

AM, MA, CE

MA

Peralta et al. 9679 p.p.

Peralta et al. 21850

Peralta \& Sores 12526 p.p.

AM, MA, CE Peralta \& Soares 12546 p.p. 
Tabela 1 (continuação)

\begin{tabular}{l} 
Táxons \\
\hline Cololejeunea manaosensis (Herzog) Herzog \\
Cololejeunea microscopica (Taylor) Schiffn. \\
Cololejeunea obliqua (Nees \& Mont.) \\
Schiffn. \\
Cololejeunea subcardiocarpa Tixier \\
Cololejeunea truncatifolia (Horik.) Mizut.
\end{tabular}

*Colura calyptryfolia (Hook.) Dummort.

Colura tenuicornis (A. Evans) Steph.

Dicranolejeunea axilaris (Nees \& Mont.) Schiffn.

Drepanolejeunea aculeata Bischl.

Drepanolejeunea anoplantha (Spruce) Steph.

Drepanolejeunea araucariae Steph.

Drepanolejeunea campanulata (Spruce) Steph.

Drepanolejeunea fragilis Bischl.

Drepanolejeunea grollei E. Reiner \& Schafer

Drepanolejeunea lichenicola (Spruce)

Steph.

Drepanolejeunea mosenii Bischl.

Frullanoides densifolia Raddi

Frullanoides liebmanniana (Lindenb \&

Gottsche) van Slageren

*Frullanoides tristis (Steph.) van Slageren

Harpalejeunea oxyphylla (Nees \& Mont.) Steph.

Harpalejeunea stricta (Lindenb. \& Gottsche) Steph.

Lejeunea asthenica Spruce

Lejeunea aphanes Spruce

Lejeunea bermudiana (A. Evans) R.M. Schust.

Lejeunea capensis Gottsche

Lejeunea caulicalyx (Steph.) E. Reiner \& Goda

Distr. Mundial

Endêmica do Brasil AM, MT, PA, SP

América tropical e África

América tropical

América tropical

Pantropical

Regiões oceânicas da

África, América Latina e leste da Europa.

Pantropical

América tropical

Endêmica do Brasil RJ, SP

Neotropical

Neotropical

Neotropical

América tropical

Endêmica do Brasil - SP

Neotropical

América tropical

América tropical

América tropical

América tropical

América tropical

América tropical

América tropical

América tropical

América tropical e África

Pantropical

América tropical
Dom. Fito.

Voucher

AM, PN, MA

Yano \& Marcelli 19483 p.p.

MA

Peralta et al. 21818

AM, MA

Peralta et al. 22074

AM, MA, CE

$\mathrm{AM}, \mathrm{CE}, \mathrm{MA}$

Peralta et al. 22016

Yano \& Prado 26103 p.p.

MA

Peralta \& Soares 12551

MA

MA

MA

AM, MA

MA

MA

AM, MA

MA

MA

AM, MA

AM, CE, MA

AM, MA, CE

MA

AM, MA

AM, MA

AM, MA

MA

AM, MA

MA

AM, CE, MA, PN Peralta \& Soares 12561 p.p.
Peralta et al. 10006 p.p.

Peralta et al. 21832 p.p.

Peralta et al. 22017

Yano \& Prado 26028

Schafer-Verwimp \& Verwimp 14463 (Isotipo)

Peralta et al. 21950

Peralta et al. 21833

Peralta et al. 9603

Peralta 12534

Peralta et al. 22115

Yano et al. 15664

Peralta \& Soares 12575

Peralta 21773

Peralta \& Soares 12569

Peralta et al. 10050

Peralta et al. 21674

continua 
Tabela 1 (continuação)

\begin{tabular}{|c|c|c|c|}
\hline Táxons & Distr. Mundial & Dom. Fito. & Voucher \\
\hline $\begin{array}{l}\text { Lejeunea cerina (Lehm. \& Lindenb.) } \\
\text { Gottsche et al. }\end{array}$ & América tropical & AM, MA & Giancotti 46 \\
\hline Lejeunea controversa Gottsche & América tropical & AM, MA & Peralta \& Soares $12561 \mathrm{p}$. \\
\hline $\begin{array}{l}\text { Lejeunea cristulaeflora (Gottsche ex Steph.) } \\
\text { E. Reiner \& Goda }\end{array}$ & América tropical & $\mathrm{MA}, \mathrm{CE}$ & Peralta \& Soares 12581 \\
\hline $\begin{array}{l}\text { Lejeunea cristulata (Steph.) M.E. Reiner } \\
\text { \& Goda }\end{array}$ & $\begin{array}{l}\text { Endêmica do Brasil - } \\
\text { BA, ES, MG, PE, PR, } \\
\text { RS, SC, SP }\end{array}$ & MA & Peralta \& Soares 12635 \\
\hline $\begin{array}{l}\text { Metalejeunea cucullata (Reinw. et al.) } \\
\text { Grolle }\end{array}$ & Neotropical & $\mathrm{MA}, \mathrm{CE}$ & Peralta et al. 21706 \\
\hline *Lejeunea diversicuspis Spruce & $\begin{array}{c}\text { Endêmica do Brasil - } \\
\text { AM, SP }\end{array}$ & $\mathrm{AM}$ & Peralta et al. 9905 \\
\hline Lejeunea flava (Sw.) Nees & Pantropical & $\begin{array}{l}\mathrm{AM}, \mathrm{CA}, \mathrm{CE}, \mathrm{MA} \\
\text { PA, PN }\end{array}$ & Peralta \& Soares 12506 \\
\hline $\begin{array}{l}\text { Lejeunea grossiretis (Steph.) E. Reiner \& } \\
\text { Goda }\end{array}$ & $\begin{array}{l}\text { Endêmica do Brasil - } \\
\text { BA, RJ, SP }\end{array}$ & MA & Peralta et al. 9422 p.p. \\
\hline $\begin{array}{l}\text { Lejeunea grossitexta (Steph.) M.E. Reiner } \\
\text { \& Goda }\end{array}$ & Neotropical & MA & Peralta \& Soares 12509 \\
\hline *Lejeunea herminieri (Steph.) R.L. Zhu & América tropical & $\mathrm{CA}$ & Peralta et al. 9426 \\
\hline $\begin{array}{l}\text { Lejeunea laeta (Lehm. \& Lindenb.) Lehm. } \\
\text { \& Lindenb. }\end{array}$ & Neotropical & MA & Yano \& Prado 26114 p.p. \\
\hline Lejeunea laetevirens Nees \& Mont. & América tropical & $\mathrm{AM}, \mathrm{CA}, \mathrm{CE}, \mathrm{MA}, \mathrm{PN}$ & Peralta et al. 9674 \\
\hline Lejeunea monimiae (Steph.) Steph. & $\begin{array}{l}\text { Brasil, Norte da } \\
\text { Argentina }\end{array}$ & MA & Yano \& Marcelli 15754 \\
\hline oclada Spruce & $\begin{array}{l}\text { Endêmica do Brasil - } \\
\text { AL, BA, CE, ES, MG, } \\
\text { PE, PR, RJ, SC, SP }\end{array}$ & MA & Peralta \& Soares 12543 p.p. \\
\hline Lejeunea phyllobola Nees \& Mont. & América tropical & $\mathrm{AM}, \mathrm{CA}, \mathrm{CE}, \mathrm{MA}, \mathrm{PN}$ & Peralta \& Soares 12550 \\
\hline $\begin{array}{l}\text { Lejeunea pterigonia (Lehm. \& Lindenb.) } \\
\text { Mont. }\end{array}$ & América tropical & MA & Gibertoni 56 \\
\hline Lejeunea puiggariana Steph. & Neotropical & MA, PN & Peralta et al. 12553 p.p. \\
\hline *Lejeunea ramulosa Spruce & $\begin{array}{l}\text { Endêmica do Brasil - } \\
\text { SC, SP }\end{array}$ & MA & Yano \& Marcelli 19479 p.p. \\
\hline Lejeunea ruthii (A. Evans) R.M. Schuster & América tropical & MA & Peralta \& Soares 12538 \\
\hline Lejeunea serpillifolioides (Raddi) Gradst. & América tropical & AM, MA & Peralta et al. 21803 \\
\hline $\begin{array}{l}\text { Leptolejeunea elliptica (Lehm. \& Lindenb.) } \\
\text { Schiffn. }\end{array}$ & América tropical & $\mathrm{AM}, \mathrm{CA}, \mathrm{CE}, \mathrm{MA}$ & Peralta et al. 22013 \\
\hline Leptolejeunea exocellata (Spruce) A. Evans & América tropical & $\mathrm{AM}, \mathrm{MA}, \mathrm{CE}, \mathrm{PN}$ & Yano \& Marcelli 19497 p.p. \\
\hline Lopholejeunea subfusca (Nees) Schiffn. & Pantropical & $\mathrm{AM}, \mathrm{MA}, \mathrm{CA}, \mathrm{CE}, \mathrm{PN}$ & Yano \& Marcelli 19542 \\
\hline Marchesinia brachiata (Sw.) Schiffn. & $\begin{array}{l}\text { América tropical e } \\
\text { África }\end{array}$ & $\mathrm{AM}, \mathrm{CE}, \mathrm{MA}$ & Peralta et al. 22059 \\
\hline $\begin{array}{l}\text { Thysananthus auriculatus (Wilson \& Hook) } \\
\text { Sukkharak \& Gradst. }\end{array}$ & América tropical & $\mathrm{AM}, \mathrm{CE}, \mathrm{MA}$ & Peralta et al. 21897 \\
\hline
\end{tabular}


Tabela 1 (continuação)

\begin{tabular}{|c|c|c|c|}
\hline Táxons & Distr. Mundial & Dom. Fito. & Voucher \\
\hline Microlejeunea bullata (Taylor) Steph. & América tropical & $\begin{array}{l}\text { AM, CA, CE, MA, } \\
\text { PA, PN }\end{array}$ & $\begin{array}{l}\text { Schafer-Verwimp \& } \\
\text { Verwimp } 6960\end{array}$ \\
\hline Microlejeunea epiphylla Bischl. & Neotropical & $\mathrm{AM}, \mathrm{CA}, \mathrm{CE}, \mathrm{MA}, \mathrm{PN}$ & Yano \& Marcelli 15687 \\
\hline Microlejeunea squarrosa J. Heinrichs et al. & $\begin{array}{l}\text { Endêmica do Brasil - } \\
\text { MG, PR, RJ, SC, SP }\end{array}$ & MA & Peralta et al. 9962 \\
\hline $\begin{array}{l}\text { Schiffneriolejeunea polycarpa (Nees) } \\
\text { Gradst. }\end{array}$ & Pantropical & $\mathrm{AM}, \mathrm{MA}, \mathrm{CA}, \mathrm{CE}, \mathrm{PN}$ & Peralta et al. 21921 \\
\hline \multicolumn{4}{|l|}{ Lepidoziaceae } \\
\hline Bazzania aurescens Spruce & América tropical & $\mathrm{AM}, \mathrm{CE}, \mathrm{MA}$ & Peralta et al. 21811 \\
\hline $\begin{array}{l}\text { *Bazzania cuneistipula (Gottsche \& Lindenb) } \\
\text { Trevis. }\end{array}$ & $\begin{array}{l}\text { Sudeste do Brasil e } \\
\text { norte dos Andes }\end{array}$ & MA & Peralta et al. 9765 \\
\hline Bazzania falcata (Lindenb.) Trevis. & América tropical & AM, MA & Vital 9673 \\
\hline $\begin{array}{l}\text { Bazzania jamaicensis (Lehm. \& lindeb.) } \\
\text { Trevis. }\end{array}$ & América tropical & MA & Yano \& Watanabe 9235 \\
\hline $\begin{array}{l}\text { Bazzania gracilis (Hampe \& Gottsche) } \\
\text { Steph. }\end{array}$ & América tropical & AM, MA & Yano \& Prado 26128 \\
\hline Bazzania hookeri (Lindenb.) Trevis. & América tropical & $\mathrm{AM}, \mathrm{MA}$ & Peralta 73 \\
\hline Bazzania nitida (F. Weber) Grolle & $\begin{array}{c}\text { Endêmica do Brasil - } \\
\text { BA, ES, MG, PE, PR, } \\
\text { RJ, RS, SC, SP }\end{array}$ & MA & Peralta et al. 21821 \\
\hline Kurzia brasililensis (Steph.) Grolle & $\begin{array}{c}\text { Endêmica do Brasil - } \\
\text { AC, AP, BA, DF, ES, } \\
\text { GO, MG, MT, RJ, RS, } \\
\text { SC, SP }\end{array}$ & $\mathrm{AM}, \mathrm{MA}, \mathrm{CE}$ & Vital 508 \\
\hline Kurzia capillaris (Sw.) Grolle & $\begin{array}{l}\text { América tropical e } \\
\text { África }\end{array}$ & $\mathrm{AM}, \mathrm{CE}, \mathrm{MA}$ & Peralta et al. 21786 \\
\hline Lepidozia brasiliensis Steph. & América do Sul & MA & Peralta et al. 21797 \\
\hline Lepidozia coilophylla Taylor & América tropical & MA & Peralta et al. 10037 \\
\hline Lepidozia cupressina (Sw.) Lindenb. & Cosmopolita & MA & Gibertoni 48 \\
\hline Lepidozia inaequalis Lehm. \& Lindenb. & América do Sul & MA & Yano \& Marcelli 19666 \\
\hline $\begin{array}{l}\text { Paracromastigum pachyrhizum (Nees) } \\
\text { Fulford }\end{array}$ & América tropical & $\mathrm{MA}, \mathrm{CE}$ & Peralta et al. 22105 \\
\hline Telaranea diacantha (Mont.) Engel \& Merr. & Pantropical & $\mathrm{AM}, \mathrm{CE}, \mathrm{MA}$ & Peralta et al. 9518 \\
\hline $\begin{array}{l}\text { Telaranea nematodes (Gottshe ex Austin) } \\
\text { M. Howe }\end{array}$ & Pantropical & $\mathrm{AM}, \mathrm{CE}, \mathrm{MA}$ & Peralta et al. 21917 \\
\hline \multicolumn{4}{|l|}{ Lophocoleaceae } \\
\hline $\begin{array}{l}\text { Chiloscyphus lindmannii (Steph.) J.J. Engel } \\
\text { \& R.M. Schust. }\end{array}$ & $\begin{array}{l}\text { América do Sul } \\
\text { tropical }\end{array}$ & $\mathrm{AM}, \mathrm{CE}, \mathrm{MA}$ & Peralta et al. 12603 \\
\hline $\begin{array}{l}\text { Chiloscyphus mandonii (Steph.) J.J. Engel } \\
\text { \& R.M. Schust. }\end{array}$ & $\begin{array}{l}\text { América do Sul } \\
\text { tropical }\end{array}$ & MA & Peralta et al. 10044 \\
\hline $\begin{array}{l}\text { Chiloscyphus martianus (Nees) J.J. Engel } \\
\text { \& R.M. Schust. }\end{array}$ & $\begin{array}{l}\text { América tropical e } \\
\text { África }\end{array}$ & $\mathrm{AM}, \mathrm{CE}, \mathrm{MA}, \mathrm{PN}$ & Peralta 9634 \\
\hline
\end{tabular}


Tabela 1 (continuação)

\begin{tabular}{|c|c|c|c|}
\hline Táxons & Distr. Mundial & Dom. Fito. & Voucher \\
\hline $\begin{array}{l}\text { Chiloscyphus muricatus (Lehm.) J.J. Engel } \\
\text { \& R.M. Schust. }\end{array}$ & Cosmopolita & MA & Peralta et al. 9522 \\
\hline Clasmatocolea vermicularis (Lehm.) Grolle & América tropical & MA, PN & Peralta et al. 9576 \\
\hline \multicolumn{4}{|l|}{ Geocalycaceae } \\
\hline Leptoscyphus amphibolius (Nees) Schiffn. & América tropical & MA, CE & Peralta et al. 9588 \\
\hline Leptoscyphus porphyrius (Nees) Grolle & América tropical & AM, MA & Peralta et al. 9554 \\
\hline Leptoscyphus spectabilis (Steph.) Grolle & América tropical & AM, MA & Peralta et al. 10013 \\
\hline \multicolumn{4}{|l|}{ Lophocoleaceae } \\
\hline $\begin{array}{l}\text { Chiloscyphus pycnophyllus (Spruce) J.J. } \\
\text { Engel \& R.M. Schust. }\end{array}$ & Subcosmopolita & $\mathrm{AM}, \mathrm{CE}, \mathrm{MA}$ & Peralta et al. 9702 \\
\hline Lophocolea bidentata (L.) Dumort. & América tropical & MA & Yano \& Marcelli 19397 \\
\hline \multicolumn{4}{|l|}{ Lunulariaceae } \\
\hline Lunularia cruciata (L.) Dumort. & Subcosmopolita & MA & Peralta et al. 21658 \\
\hline \multicolumn{4}{|l|}{ Marchantiaceae } \\
\hline Dumortiera hirsuta (Sw.) Nees & América tropical & AM, CE, MA, PN & Yano 318 \\
\hline *Marchantia breviloba A. Evans & $\begin{array}{l}\text { América do Sul } \\
\text { tropical }\end{array}$ & MA & Vital 577 \\
\hline Marchantia chenopoda L. & América tropical & AM, CE, MA, PN & Peralta et al. 21777 \\
\hline \multicolumn{4}{|l|}{ Metzgeriaceae } \\
\hline Metzgeria acuminada Steph. & América tropical & MA & Yano \& Marcelli 15700 \\
\hline Metzgeria agnewiae Kuwah. & América tropical & MA & Yano \& Marcelli 15701 \\
\hline Metzgeria albinea Spruce & Pantropical & $\mathrm{CE}, \mathrm{MA}$ & Peralta et al. 21845 \\
\hline Metzgeria bahiensis Schiffn. & $\begin{array}{l}\text { Endêmica do Brasil - } \\
\text { BA, RS, SP }\end{array}$ & MA & Peralta et al. 22088 \\
\hline Metzgeria conjugata Lindb. & Subcosmopolita & MA & Peralta et al. 9915 \\
\hline Metzgeria cratoneura Schiffn. & $\begin{array}{l}\text { Endêmica do Brasil - } \\
\text { PR, RJ, RS, SC, SP }\end{array}$ & MA & Peralta et al. 9896 \\
\hline Metzgeria furcata (L.) Corda & Subcosmopolita & $\mathrm{AM}, \mathrm{MA}, \mathrm{CE}$ & Yano \& Marcelli 19619 \\
\hline *Metzgeria hegewaldii Kuwah. & América tropical & MA & Peralta et al. 9587 \\
\hline Metzgeria leptoneura Spruce & América tropical & AM, MA & Peralta 83 p.p. \\
\hline Metzgeria scyphigera A. Evans & América tropical & MA & Peralta et al. 21662 \\
\hline Metzgeria subaneura Schiffn. & $\begin{array}{l}\text { Endêmica do Brasil - } \\
\text { AC, ES, MG, PR, RJ, } \\
\text { RS, SP }\end{array}$ & $\mathrm{AM}, \mathrm{MA}$ & Marcelli \& Yano 12335 p.p. \\
\hline Metzgeria uncigera A. Evans & América tropical & MA & Peralta 188 \\
\hline \multicolumn{4}{|l|}{ Monocleaceae } \\
\hline Monoclea gottschei Lindb. & América tropical & AM, MA & Yano \& Marcelli 19631 \\
\hline \multicolumn{4}{|l|}{ Pelliaceae } \\
\hline $\begin{array}{l}\text { Noteroclada confluens Taylor ex Hook. \& } \\
\text { Wilson }\end{array}$ & Neotropical & MA, CE & Vital 576 \\
\hline \multicolumn{4}{|l|}{ Pallaviciniaceae } \\
\hline *Jensenia difformis (Nees) Grolle & América tropical & MA & Yano 319 p.p. \\
\hline
\end{tabular}


Tabela 1 (continuação)

\begin{tabular}{|c|c|c|c|}
\hline Táxons & Distr. Mundial & Dom. Fito. & Voucher \\
\hline Pallavicinia lyelli (Hook.) S.F. Gray & América tropical & $\mathrm{AM}, \mathrm{CE}, \mathrm{MA}, \mathrm{PN}$ & Visnadi \& Vital 2356 \\
\hline Symphyogyna aspera Steph. & América tropical & $\mathrm{AM}, \mathrm{CE}, \mathrm{MA}, \mathrm{PN}$ & Peralta \& Soares 12530 \\
\hline $\begin{array}{l}\text { Symphyogyna brasiliensis (Nees) Nees \& } \\
\text { Mont. }\end{array}$ & Neotropical & $\mathrm{AM}, \mathrm{CE}, \mathrm{MA}$ & Vital 14600 \\
\hline Symphyogyna brongniarti Mont. & América tropical & $\mathrm{AM}, \mathrm{MA}$ & Peralta et al. 9744 \\
\hline $\begin{array}{l}\text { Symphyogyna podophylla (Thunb.) Mont. } \\
\& \text { Nees }\end{array}$ & $\begin{array}{c}\text { América tropical e } \\
\text { África }\end{array}$ & MA & Vital 9648 \\
\hline \multicolumn{4}{|l|}{ Plagiochilaceae } \\
\hline Plagiochila adianthoides (Sw.) Lindenb. & América tropical & $\mathrm{MA}, \mathrm{CE}$ & Peralta et al. 21813 \\
\hline Plagiochila bifaria (Sw.) Lindenb. & América tropical & AM, MA & Peralta 92 \\
\hline Plagiochila corrugata (Nees) Nees \& Mont. & América tropical & $\mathrm{AM}, \mathrm{CE}, \mathrm{MA}$ & Peralta et al. 9959 \\
\hline Plagiochila cristata (Sw.) Lindenb. & América tropical & AM, MA & Vital 9681 \\
\hline *Plagiochila deflexirama Taylor & América tropical & MA & \\
\hline Plagiochila exigua (Taylor) Taylor & $\begin{array}{l}\text { América tropical e } \\
\text { África }\end{array}$ & MA & Peralta et al. 9501 \\
\hline $\begin{array}{l}\text { Plagiochila gymnocalycina (Lehm. \& } \\
\text { Lindenb.) Mont. }\end{array}$ & América tropical & MA & Peralta et al. 9754 \\
\hline Plagiochila macrostachya Lindenb. & América tropical & AM, MA & Peralta 197 \\
\hline Plagiochila montagnei Nees & América tropical & $\mathrm{AM}, \mathrm{MA}$ & Yano \& Marcelli 19412 \\
\hline $\begin{array}{l}\text { Plagiochila patula (Sw.) Nees \& Mont. ex } \\
\text { Lindenb. }\end{array}$ & América tropical & AM, MA & Peralta et la. 9981 p.p. \\
\hline Plagiochila rutilans Lindenb. & América tropical & $\mathrm{AM}, \mathrm{CE}, \mathrm{MA}$ & Peralta et al. 9625 \\
\hline Plagiochila simplex (Sw.) Lindenb. & América tropical & $\mathrm{AM}, \mathrm{CE}, \mathrm{MA}$ & Peralta et al. 9533 \\
\hline \multicolumn{4}{|l|}{ Porellaceae } \\
\hline Porella brasiliensis (Raddi) Schiffn. & $\begin{array}{l}\text { América do Sul } \\
\text { tropical }\end{array}$ & $\mathrm{MA}, \mathrm{CE}$ & Peralta et al. 9594 \\
\hline Porella swartziana (Weber) Trevis. & América tropical & $\mathrm{MA}, \mathrm{CE}$ & Peralta et al. 21660 \\
\hline \multicolumn{4}{|l|}{ Radulaceae } \\
\hline Radula angulata Steph. & América tropical & MA, CE & Yano \& Marcelli 19652 \\
\hline Radula brasilica Yamada & $\begin{array}{c}\text { Endêmica do Brasil - } \\
\text { RJ, SP }\end{array}$ & MA & Yano \& Marcelli 15718 \\
\hline Radula decora Gottsche ex Steph. & América tropical & MA & Peralta et al. 21684 \\
\hline Radula fendleri Gottsche ex Steph. & $\begin{array}{l}\text { América do Sul } \\
\text { tropical }\end{array}$ & MA & Peralta et al. 10071 \\
\hline Radula javanica Gottsche & Cosmopolita & $\mathrm{AM}, \mathrm{CE}, \mathrm{MA}, \mathrm{PN}$ & Yano et al. 22108 \\
\hline Radula kegelii Gottsche ex Steph. & América tropical & $\mathrm{AM}, \mathrm{CE}, \mathrm{MA}, \mathrm{PN}$ & Peralta 78 \\
\hline Radula mexicana Lindenb. & $\begin{array}{l}\text { América tropical, } \\
\text { África e Europa }\end{array}$ & AM, MA & Yano \& Marcelli 19673 \\
\hline Radula nudicaulis Steph. & $\begin{array}{l}\text { América do sul } \\
\text { tropical e Macronésia }\end{array}$ & MA & Peralta 187 \\
\hline Radula obovata Castle & América tropical & AM, MA & Peralta et al. 21757 \\
\hline Radula quadrata Gottsche & Cosmopolita & AM, MA & Peralta et al. 10020 \\
\hline
\end{tabular}


Tabela 1 (continuação)

\begin{tabular}{|c|c|c|c|}
\hline Táxons & Distr. Mundial & Dom. Fito. & Voucher \\
\hline Radula recubans Taylor & América tropical & AM, MA & Peralta et al. 21847 \\
\hline Radula schaefer-verwimpii K. Yamada & América tropical & MA & $\begin{array}{l}\text { Schafer-Verwimp \& } \\
\text { Verwimp } 11025\end{array}$ \\
\hline Radula sinuata Gottsche ex Steph. & América tropical & MA & Yano \& Marcelli 19474 \\
\hline Radula tenera Mitt. ex Steph. & $\begin{array}{l}\text { América do Sul } \\
\text { tropical }\end{array}$ & MA & Yano \& Prado 26039 \\
\hline Radula varilobula Castle & $\begin{array}{l}\text { América do Sul } \\
\text { tropical }\end{array}$ & Ma & Gibertoni 52 \\
\hline Radula voluta Taylor ex Gottsche & $\begin{array}{l}\text { América tropical, } \\
\text { África e Europa }\end{array}$ & MA & Peralta et al. 9534 \\
\hline \multicolumn{4}{|l|}{ Ricciaceae } \\
\hline Riccia paranaensis Hässel & América tropical & MA, PA, PN & Yano \& Watanabe 9213 \\
\hline \multicolumn{4}{|l|}{ Scapaniaceae } \\
\hline Scapania portoricensis Hampe \& Gottsche & América tropical & AM, MA & Peralta et al. 21756 \\
\hline \multicolumn{4}{|l|}{ Trichocoleaceae } \\
\hline Leiomitria brevifissa Steph. & América tropical & MA & Peralta et al. 21798 \\
\hline $\begin{array}{l}\text { Leiomitria flaccida (Spruce) J.B. Jack \& } \\
\text { Steph. }\end{array}$ & América tropical & MA & Peralta et al. 9694 \\
\hline Leiomitria tomentosa (Sw.) Gottsche & América tropical & MA & Vital 9616 \\
\hline
\end{tabular}

Tabela 2. Número de espécies encontradas no Parque Estadual de Campos do Jordão (PECJ) em relação com outras regiões. Valores entre parênteses representam a porcentagem de espécies do PECJ em relação ao total de espécies encontradas na região comparada.

Table 2. Number of the species found in the Parque Estadual de Campos do Jordão (PECJ) in relation to other regions. Values in parentheses represent the percentage of species in the PECJ in relation to the total of species found in the compared region.

\begin{tabular}{lc}
\hline Região & Diversidade de briófitas \\
\hline Parque estadual Campos do Jordão & 490 spp., dados apresentados nesse estudo \\
Estado de São Paulo & 900 spp. (54.44\%), Costa \& Peralta (2015) \\
Brasil & 1544 spp. (31,74\%), Flora do Brasil (2020, em prep.) \\
América Tropical & 3980 spp. (13\%), Gradstein et al. (2001) \\
\hline
\end{tabular}

Peralta, 2015a) e este estudo expõem o quanto o bioma é diverso (das 490 spp. 482 espécies ocorrem na Mata Atlântica) e as mesmas famílias citadas acima são as mais representativas neste bioma. Outros trabalhos realizados com o grupo de briófitas também encontram as mesmas famílias sendo as mais representativas (por exemplo, Costa et al. 2015a, Silva \& Porto 2015, Carmo et al. 2016, Carmo et al. 2018).

Para as hepáticas a família Lejeuneaceae se destaca, apresentando 80 espécies, o que representa $35 \%$ das hepáticas encontradas nessa área. A família Lejeuneaceae aparece em inúmeros trabalhos como sendo a mais diversa entre as hepáticas para o Estado de São Paulo (Visnadi 2005, Visnadi 2009, Yano \& Peralta 2007, Peralta \& Yano 2008, Carmo et al. 2016,
2018) e para as demais áreas do Brasil (Valente \& Pôrto 2006, Santos \& Costa 2008, Costa et al. 2011, Reis et al. 2015, Silva \& Pôrto 2015 e Carmo \& Peralta 2016). Para o Brasil, estudos apontam que existam aproximadamente 285 espécies de Lejeuneaceae, distribuídos em 55 gêneros (Costa \& Peralta 2015a, b) e a Mata Atlântica é o domínio fitogeográfico com o maior número de espécies (246 spp., Flora do Brasil 2020, em prep.), principalmente por conta do clima úmido e pôr uma infinidade de substratos onde as espécies podem se desenvolver como corticícolas, epífitas, rupícolas ou terrícolas (Gradstein et al. 2001).

$\mathrm{Na}$ área do parque os antóceros estão representados pelas famílias Dendrocerotaceae (Nothoceros minarum (Nees) J. C. Villarreal), Notothyladaceae (Phaeoceros 
laevis (L.) Prosk.) e Phymatocerotaceae Duff et al. (Phymatoceros bulbiculosus (Broth.) Stotler, W.T. Doyle \& Crand-Stotl.). Essas espécies são encontradas se desenvolvendo sobre rochas próximas da água e em barrancos úmidos, geralmente associadas a espécies de musgos (Fissidentaceae e Pottiaceae).

Os dados apresentados nesse trabalho acrescentaram importantes informações sobre a ocorrência, diversidade e distribuição da brioflora na Mata Atlântica para o Estado de São Paulo. O PECJ apresentou 1/3 da diversidade de briófitas estimada para o Brasil com um número alto de espécies endêmicas (63 spp.), dentre estas Lejeunea ramulosa Spruce é uma nova ocorrência para o Estado de São Paulo. Ao todo foram encontradas 26 novas ocorrências para o Estado de São Paulo, destas 20 espécies ocorrem na América Tropical, quatro são neotropicais, uma espécie (Leiomela bartramioides (Hook.) Paris) é Pantropical e outra espécie (Colura calyptryfolia (Hook.) Dummort.) era conhecida apenas para regiões oceânicas da África, América Latina e Leste da Europa.

A Mata Atlântica é o domínio fitogeográfico com maior riqueza de espécies de briófitas no país (Costa et al. 2011) e estudos como esse, mostram a importância de sua conservação. O PECJ e outras unidades de conservação, garantem a proteção dessas áreas e reforçam a importância da conservação, preservando a diversidade vegetal no Estado de São Paulo (Visnadi 2005). Baseado nos resultados desse estudo, das 409 espécies encontradas na área do paque, 203 espécies (ca. 41\%) tem ocorrência exclusiva na Mata Atlântica, o que ressalta a importância desse domínio fitogeográfico.

\section{Agradecimentos}

Ao corpo administrativo do Parque Estadual de Campos do Jordão pela recepção.

\section{Literatura citada}

ALMEIDA, F.F.M. 1974. Fundamentos geológicos do relevo paulista. Boletim do Instituto de Geografia. São Paulo. Instituto de Geografia.

Bordin, J. \& Yano, O. 2013. Fissidentaceae (Bryophyta) do Brasil. Boletim do Instituto de Botânica 22: 1-72.

Buck, W.R. 1998. Pleurocarpous Mosses of the West Indies. Memoirs of The New York Botanical Garden 1: 1-401.

Câmara, P.E.A.S. 2008a. Musgos pleurocárpicos das matas de galeria da Reserva Ecológica do IBGE, RECOR, Distrito Federal, Brasil. Acta Botanica Brasilica 22: 573-581.
Câmara, P.E.A.S. 2008b. Musgos acrocárpicos das Matas de Galeria da Reserva Ecológica do IBGE, RECOR, Distrito Federal, Brasil. Acta Botanica Brasilica 22: 1027-1035.

Câmara, P.E.A.S. \& Costa, D.P. 2006. Hepáticas e antóceros das matas de galeria da Reserva Ecológica do IBGE, RECOR, Distrito Federal, Brasil. Hoehnea 33: 79-87.

Carmo, D.M. \& Peralta, D.F. 2016. Survey of bryophytes in Serra da Canastra National Park, Minas Gerais, Brasil. Acta Botanica Brasilica 30: 254-265.

Carmo, D.M., Lima, J.S., Amélio, L.A. \& Peralta, D.F. 2016. Briófitas do Parque Estadual da Serra do Mar, Núcleo de Santa Virgínia, Estado de São Paulo, Brasil. Hoehnea 43: 265-287.

Carmo, D.M., Lima, J.S., Silva, M. I., Amélio, L.A. \& Peralta, D.F. 2018. Briófitas da Reserva Particular do Patrimônio Natural da Serra do Caraça, Estado de Minas Gerais, Brasil. Hoehnea 45: 484-508.

Carvalho-Silva, M., Stech, M., Soares-Silva, L.H., Buck, W.R., Wickett, N.J., Liu, Y., \& Câmara, P.E.A.S. 2017. A molecular phylogeny of the Sematophyllaceae s.l. (Hypnales) based on plastid, mitochondrial and nuclear markers, and its taxonomic implications. Taxon 66: 811-831.

Costa, D.P. \& Lima, F.M. 2005. Moss diversity in the tropical rainforests of Rio de Janeiro, Southeastern Brazil. Revista Brasileira de Botânica 28: 671-685.

Costa, D.P. \& Santos, N.D. 2009. Conservação de hepáticas na Mata Atlântica do sudeste do Brasil: uma análise regional no Estado do Rio de Janeiro. Acta Botanica Brasilica 23: 913-922.

Costa, D.P. 2010. Briófitas. In: R.C. Forzza, J.F. Baumgratz, C.E. de M. Bicudo, D. Canhos, A.A. Carvalho Jr., A. Costa, D.P. Costa, M. Hopkins, M.P. Leitman, L.G. Lohmann, E.N. Lughadha, L.C. Maia, G. Martinelli, M. Menezes, M.P. Morin, M. Nadruz, A.L. Peixoto, J.R. Pirani, J. Prado, L.P. Queiroz, S. de Souza, V.C. Souza, J.R. Stehmann, L.S. Sylvestre, B.M.T. Walter \& D.C. Zappi (orgs.). Catálogo de plantas e fungos do Brasil, v. 1,1 ed. Instituto de Pesquisas Jardim Botânico do Rio de Janeiro, Rio de Janeiro, pp. 452-521.

Costa, D.P., Pôrto, K.C., Luizi-ponzo, A.P., Ilkiu-Borges, A.L., Bastos, C.J.P., Câmara, P.E.A.S., Peralta, D.F., Bôas-Bastos, S.B.V., Imbassahy C.A.A., Henriques, D.K., Gomes, H.C.S., Rocha, L.M., Santos, N.D., Siviero, T. S., Vaz- Imbassahy, T.F. \& Churchill, S.P. 2011. Synopsis of the Brazilian moss flora: checklist, distribution and conservation. Nova Hedwigia 93: 277-334.

Costa, D.P. \& Peralta, D.F. 2015a. Bryophytes diversity in Brazil. Rodriguésia 66: 1-9.

Costa, D.P. \& Peralta, D.F. 2015b. Briófitas. In: Lista de Espécies da Flora do Brasil. Jardim Botânico do Rio de Janeiro. Disponível em http://floradobrasil.jbrj.gov.br/ jabot/floradobrasil/FB128472 (acesso em 21-II-2017). 
Crandall-Stotler, B., Stotler, R.E. \& Long, D.G. 2009. Morphology and classification of the Marchantiophyta. In: B. Goffinet \& A.J. Shaw, 2 ed. Bryophyte Biology. Cambridge, Cambridge University Press., pp. 1-54.

Frahm, J.P. 1991. Dicranaceae: Campylopodioideae, Paraleucobryoideae. Flora Neotropica Monograph 54: 1-237.

Frey W, Stech M. 2009. Marchantiophyta, Bryophyta, Anthocerotophyta. In: W. Frey, M. Stech \& E. Fischer. Syllabus of Plant Families - Engler's Syllabus der Pflanzenfamilien, 13 ed. Part 3: Bryophytes and seedless Vascular Plants. Frey W, editor. Borntraeger, Berlin, pp. 13-115.

FUNDAÇÃO SOS MATAATLÂNTICA. 2017. Florestas. A Mata Atlântica. Disponivel em https://www.sosma. org.br/nossa-causa/a-mata-atlantica/ (acesso em 20-II-2017).

Goffinet, B., Buck, W.R. \& Shaw, A.J. 2009. Morphology, anatomy and classification of the Bryophyta. In: B. Goffinet \& A.J. Shaw, 2 ed. Bryophyte Biology. Cambdrige University Press, pp. 56-138.

Gradstein, S.R., Churchill, S.P. \& Salazar-Allen, N. 2001. Guide to the Bryophytes of Tropical America. Memoirs of The New York Botanical Garden 86: 1-577.

Gradstein, S.R. \& Costa, D.P. 2003. The Hepaticae and Anthocerotae of Brazil. Memoirs of The New York Botanical Garden 87: 1-318.

Gradstein, S.R. 2015. Annotated key to the species of Plagiochila (Marchantiophyta) from Brazil. Pesquisas, Botânica 67: 23-36.

Gradstein, S.R. \& Ilkiu-Borges, A.L. 2015. A taxonomic revision of the genus Odontoschisma (Marchantiophyta: Cephaloziaceae). Nova Hedwigia 100: 15-100.

Hallingbäck, T. \& Hodgetts, N.G. 2000. Mosses, liverworts \& hornworts. Status survey and conservation action plan for bryophytes. IUCN/SSC Bryophyte Specialist Group. IUCN, Gland, Switzerland and Cambridge, UK.

Hallingbäck, T. \& Tan, B.C. 2010. Past and present activities and future strategy of bryophyte conservation. Phytotaxa 9: 266-274.

IBGE (Instituto Brasileiro de Geografia e Estatística). 2012. Manual técnico da vegetação brasileira. Série: Manuais técnicos em geociências. Fundação Instituto Brasileiro de Geografia e Estatística, Rio de Janeiro.

Jarenkow, J.A. \& Baptista, L.R.M. 1987. Composição florística e estrutura da mata com Araucária na Estação Ecológica de Aracuri, Esmeralda, RS. Napaea, Porto Alegre, v.3, pp. 9-18.

Kruijer, J.D. 2002. Hypopterygiaceae of the world. Blumea. Supplement 13: 1-388.
Longton, R.E. 1992. The role of bryophytes and lichens in terrestrial Ecosystems. In: J.W. Bates, \& A.M. Farmer (eds.). Bryophytes and lichens in a changing environment. Oxford University Press, New York, pp. 32-76.

Moreira, M., Baretta, D., Tsai, S.M. \& Cardoso, E.J.B.N. 2006. Spore density and root colonization by arbuscular mycorrhizal fungi in preserved or disturbed Araucaria angustifolia (Bert.) O. Ktze. ecosystems. Scientia Agrícola, v. 63, 4: 380-385.

Peralta, D.F. \& Yano, O. 2008. Briófitas do Parque Estadual da Ilha Anchieta, Ubatuba, estado de São Paulo, Brasil. Iheringia 63: 101-127.

Peralta, D.F. \& Yano, O. 2012. Briófitas da Serra do Itapeti. In: M.S.C. Morini \& V.F.O. Miranda (org.). Serra do Itapeti: Aspectos Históricos, Sociais e Naturalísticos. Viena Gráfica e Editora, Santa Cruz do Rio Pardo v. 1. pp. 1-397.

Puttick, M.N., Morris, J.L., Williams, T.A., Cox, C.J., Edwards, D., Kenrick, P., Pressel, S., Wellman, C.H., Schneider, H., Pisani, D. \& Donoghue, P.C.J. 2018. The Interrelationships of Land Plants and the Nature of the Ancestral Embryophyte. Current Biology 28: 733-745.

Reis, L.C., Oliveira, H.C. \& Bastos, C.J.P. 2015. Hepáticas (Marchantiophyta) epífitas de duas áreas de floresta atlântica no Estado da Bahia, Brasil. Pesquisas, Botânica 67: 225-241.

Renzaglia, K.S., Villarreal, J.C. \& Duff, R.J. 2009. New insights into morphology, anatomy and systematics of hornworts. In: B. Goffinet \& , A.J. Shaw, 2 ed. Bryophyte Biology. Cambridge, Cambridge University Press. pp. 139-171.

Santos, N.D. \& Costa, D.P. 2008. A importância de Reservas Particulares do Patrimônio Natural para a conservação da brioflora da Mata Atlântica: um estudo em El Nagual, Magé, RJ, Brasil. Acta Botanica Brasilica 22: 359-372.

Santos, N.D. \& Costa, D.P. 2010. Phytogeography of the liverwort flora of the Atlantic Forest of southeastern Brazil. Journal of Bryology 32: 9-22.

Seibert, C.L., Negreiros, O.C., Bueno, R.A., Emerich, W., Moura-Netto, B.V., Marcondes, M.A.P, Cesar, S.F., Guillanimon, J.R., Montagna, R.A.A., Barreto, J.R., Oliveira, M.C. \& Godoi, A. 1975. Plano de manejo no Parque Estadual de Campos de Jordão. Boletim Técnico do Instituto Florestal de São Paulo 19: 1-153.

Silva, M.P.P. \& Pôrto, K.C. 2015. Diversity of bryophytes in priority areas for conservation in the Atlantic Forest of northeast Brazil. Acta Botanica Brasilica 29: 16-23.

Sharp, A.J., Crum, H. \& Eckel, P. 1994. The Moss Flora of Mexico. Memoirs of The New York Botanical Garden 69: 1-1113. 
Souza, R.P.M. 2008. Estrutura da comunidade arbórea de trechos de florestas de Araucária no estado de São Paulo, Brasil. Dissertação de Mestrado, Escola Superior de Agricultura Luiz de Queiroz, Universidade de São Paulo, Piracicaba.

Södeström, L., Crandall-Stotler, B., Stotler, R.E., Vána, J., Hagborg, A. \& Konrat, M.V. 2013a. Notes on Early Land Plants Today. 36. Generic treatment of Lophocoleaceae (Marchantiophyta). Phytotaxa 97: 36-43.

Södeström, L., Vána, J., Crandall-Stotler, B., Stotler, R.E., Hagborg, A. \& Konrat, M.V. 2013b. Notes on Early Land Plants Today. 43. New Combinations in Lophocoleaceae (Marchantiophyta). Phytotaxa 112: 18-32.

Turetsky, M.R. 2003. The Role of Bryophytes in Carbon and Nitrogen Cycling. The Bryologist 106: 395-409.

Visnadi, S.R. 2005. Brioflora da Mata Atlântica do estado de São Paulo: região norte. Hoehnea 32: 215-231.
Visnadi, S.R. 2006. Sematophyllaceae da Mata Atlântica do nordeste do Estado de São Paulo. Hoehnea 33: 455-484.

Visnadi, S.R. 2009. Briófitas do Caxetal, em Ubatuba, São Paulo, Brasil. Tropical Bryology 30: 8-14.

Visnadi, S.R. 2013. Briófitas de áreas antrópicas do Parque Estadual da Serra do Mar, Núcleo Picinguaba, Ubatuba, estado de São Paulo, Brasil. Boletim do Museu Paraense Emílio Goeldi 8: 49-62.

Yano, O. \& Peralta, D.F. 2007. Briófitas da Ilha do Bom Abrigo, Estado de São Paulo, Brasil. Hoehnea 34: 87-94.

Yano, O. \& Peralta, D.F. 2008. Briófitas da Ilhabela, Estado de São Paulo, Brasil. Hoehnea 35: 111-121.

Yano, O. \& Peralta, D.F. 2009. Flora de Grão-Mogol, Minas Gerais. Briófitas (Bryophyta e Marchantiophyta). Boletim de Botânica da Universidade de São Paulo 27: 1-26.

Yano, O. \& Peralta, D.F. 2011. Bryophytes from Serra de São José, Tiradentes, Minas Gerais, Brasil. Boletim de Botânica da Universidade de São Paulo 21: 141-172. 